\title{
Information Acquisition and Diffusion in Markets*
}

\author{
Atabek Atayev $^{\dagger}$ and Maarten Janssen ${ }^{\ddagger}$ \\ Friday $1^{\text {st }}$ October, 2021
}

\begin{abstract}
Consumers can acquire information through their own search efforts or through their social network. Information diffusion via word-of-mouth communication leads to some consumers free-riding on their "friends" and less information acquisition via active search. Free-riding also has an important positive effect, however, in that consumers that do not actively search themselves are more likely to be able to compare prices before purchase, imposing competitive pressure on firms. We show how market prices depend on the characteristics of the network and on search cost. For example, if the search cost becomes small, price dispersion disappears, while the price level converges to the monopoly level, implying that expected prices are decreasing for small enough search cost. More connected societies have lower market prices, while price dispersion remains even in fully connected societies.
\end{abstract}

JEL Classification: D43, D83, D85

Keywords: Consumer Search; Word-of-Mouth Communication; Social Networks.

* We thank seminar participants at the Vienna Graduate School of Economics, EARIE 2017, EEA-ESEM 2018, and the 2018 Consumer Search and Switching Costs Workshop especially Daniel Garcia, Eeva Mauring, Mariya Teteryatnikova, Sandro Shelegia, John Vickers and Chris Wilson for helpful suggestions and comments on earlier versions of the paper. Atayev acknowledges financial support from the uni:docs Fellowship Programme. Janssen acknowledges financial support from the Austrian Science Foundation FWF under project number I 3487.

$\dagger$ ZEW-Leibniz Centre for European Economic Research in Mannheim. Email: atabek.atayev@zew.de

$\ddagger$ Department of Economics, University of Vienna, National Research University Higher School of Economics and CEPR. Email: maarten.janssen@univie.ac.at 


\section{Introduction}

Decentralized markets rely on how information that is dispersed over many individuals is diffused (Hayek (1945)). Individual agents are, however, not endowed with a natural amount of information. Often, they have to spend resources, such as time, to search and acquire information. Accordingly, agents will only acquire information if the expected benefits exceed the opportunity cost of doing so. This has led Grossman and Stiglitz (1980) to pose that efficient markets cannot exist if arbitrage is costly. Information can, however, also be acquired in less costly ways, namely through word-of-mouth (WOM) communication via friends (see, e.g., Ellison and Fudenberg (1995) and Campbell (2013)). ${ }^{1}$ WOM communication may come with a delay, however, as one has to wait for friends to communicate their information.

Costly information acquisition and diffusion (WOM communication) are clearly related. When few people acquire information themselves, little information will be diffused, while if information is disseminated efficiently, people may not have the incentive to spend resources to acquire information themselves. Thus, it is important to understand the interaction between the incentives to acquire information and the efficiency of the information diffusion process. This is especially so for online markets and online interaction through social networks, such as Facebook or LinkedIn. It is well documented ${ }^{2}$ that online technologies have significantly reduced the search cost related to information acquisition and increased the possibilities of diffusion and it is important to understand how these developments affect market outcomes.

In this paper we study the interaction between information acquisition, diffusion and market power, and explain the impact of changes in the connectedness of people (impacting diffusion of information through WOM) and search costs on market outcomes. We adopt a simple theoretical framework of a homogeneous goods market where firms set prices and consumers engage in costly sequential search to acquire information about prices before buying one unit of the good (Diamond (1971) and Stigler (1961)). ${ }^{3}$ Con-

\footnotetext{
${ }^{1}$ Katz and Lazarsfeld (1955) is the classic study showing that information acquired through personal contacts is the prime reason why people buy a product.

${ }^{2}$ See, e.g., Brown and Goolsbee (2002), Jensen (2007) Aker (2010), and Aker and Mbiti (2010). On word-of-mouth communication, see, e.g., Godes and Mayzlin (2004), Chen et al. (2011) and Seiler et al. (2019).

${ }^{3}$ Undoubtedly, in real world markets consumers also exchange information about product character-
} 
sumers that have searched for prices spread this information through their network. The environment we study allows us to consider the impact of social networks and search costs on information acquisition and market power. In our model, a network is characterized by two features: (i) the number of friends different consumers are connected to, and (ii) the speed with which information flows through the network.

We find that independent of the acquisition (search) cost there always exists a no-trade equilibrium that is similar to the Diamond paradox: as no one will acquire information if firms set very high prices and setting high prices is optimal if no one acquires information. ${ }^{4}$ Importantly, WOM resolves the Diamond paradox in that it creates additional equilibria with positive sales. in all of these equilibria, searching consumers follow a reservation price strategy and firms do not price above this reservation price. Under WOM, consumers determine whether or not to acquire information themselves. The possibility to get information through their social network implies that in any equilibrium it cannot be the case that all consumers acquire information themselves. If that were the case, prices would be equal to the willingness to pay and consumers would not be willing to spend a positive search cost so that free riding on also allows them to buy, possibly at the lowest price charged in the market without incurring the search cost. Thus, an endogenously determined fraction of consumers is informed through friends. As by free-riding on the information acquisition of friends the non-searching consumers will be informed with positive probability about different prices, it has an important positive effect on market competition.

In terms of the impact of the social network structure, we show that, contrary to what one may expect, even when the network gets very dense and many consumers have many connections, prices do not converge to marginal cost and price dispersion remains. What matters for price dispersion and also for the expected market price is the relative fraction of consumers that is informed of only one price. This fraction is endogenously determined and remains positive even in dense networks as information from friends comes with a delay creating an incentive to search and searching consumers buy immediately after acquiring information.

istics, such as quality, appearance and/or convenience. Yet, people also share information online about prices. In homogeneous goods markets, price communication is the only thing that matters.

${ }^{4} \mathrm{~A}$ no trade equilibrium exists in many simultaneous and sequential search models where the first search is costly for all consumers (see, e.g., Burdett and Judd (1983) and Diamond (1971)) 
The speed of information diffusion in networks is important in that it is a key determinant of the cost associated with waiting for information through WOM. We show that a higher speed of information diffusion has two opposing effects on market competition. First, it has a direct positive effect as more consumers do not search themselves, making price comparisons more likely. There is also an indirect effect, however, namely that as prices and price dispersion decline, consumers have more incentives to become active searchers themselves, especially when the speed of information diffusion is low to begin with. These two effects lead both the fraction of active consumers and firms' profits to have an inverted U-shape with respect to the speed of information diffusion: when this speed is low to begin with, firms have an incentive to increase it as this will also speed up their sales, but when the speed is already relatively large, the effect on expected price dominates and firms want to slow down information diffusion.

The impact of search cost is best illustrated by considering the case where the search cost becomes arbitrarily small. We show that in this case price dispersion disappears and almost all consumers become active themselves and buy immediately after they have searched themselves. As almost no consumer makes price comparisons, prices converge to monopoly levels. When the search cost increases, more consumers remain passive so that a larger fraction of consumers make price comparisons, resulting in lower prices. Thus, and contrary to common wisdom, prices are decreasing in search cost when WOM plays a role.

Our paper provides a new argument to overcome the Diamond paradox. ${ }^{5}$ Wolinsky (1986) resolves the Diamond paradox by having firms produce heterogeneous products and consumers searching not only for price bu also for a good product match. Stahl (1989) imposes search cost heterogeneity among consumers, where some exogenously determined fraction of "shoppers" have zero search cost and compare all prices before buying. Unlike these two papers, we endow consumers with the possibility to acquire information through WOM in addition to their own information acquisition by means of search. In this way, we endogenize the fraction of price comparing consumers and resolve the Diamond paradox.

Galeotti (2010) also combines WOM communication and consumer search. There are

\footnotetext{
${ }^{5}$ If individual consumers have downward sloping demand (or the first search is somehow free), then the Diamond paradox takes on a somewhat different form, namely that all firms charge the monopoly price.
} 
three main modeling differences between his paper and ours. First, in Galeotti (2010) consumers search for prices in a non-sequential fashion, whereas we have a sequential search framework. In most consumer retail markets, consumers observe the price at a firm before they decide whether to search another firm, making the sequential search paradigm more relevant. Second, Galeotti (2010) assumes the first search is free so that all consumers know at least one price, whereas we have truly costly search. Finally, where all consumers have the same number of links in the basic model studied in Galeotti (2010), we model the social network as a random graph. These differences in modeling lead to pronounced differences in our understanding of information acquisition and diffusion in markets. First, in our setting prices are increasing in search cost and tend to the monopoly price when search cost tends to zero, whereas in Galeotti prices converge to their competitive levels. Sequential search is the main reason for this difference. Second, if people get better connected and the network becomes dense, we show that price dispersion and market power remain, whereas in Galeotti (2010) prices converge to marginal cost. Costly first search is responsible for this important difference. ${ }^{6}$ Third, our result that free-riding has a positive impact on prices is not present in Galeotti (2010) as under non-sequential search with the first search being free, searching consumers are always better informed than non-searching consumers. ${ }^{7}$ Fourth, our modeling of search and communication through a network allows us to consider the impact of the speed with which communication travels through the network. Finally, we offer a new resolution to the Diamond paradox.

There is also a growing literature on how WOM communication affects the pricing and advertising policy of firms in the market. Earlier papers in this literature (see, e.g., Arbatskaya and Konishi (2016), Biyalogorsky et al. (2001), Bloch (2016), Campbell (2013), Chuhay (2015), Fainmesser and Galeotti (2016), Galeotti and Goyal (2009), Jun and Kim (2008), and Kornish and Li (2010)) consider how a monopoly firm can introduce its product optimally through a network assuming that consumers passively

\footnotetext{
${ }^{6}$ Janssen et al. (2005) were the first to study the impact of the first search being costly on the participation of consumers in the marketplace. In their setting (and in contrast to ours), search is, however, the only source of information acquisition.

${ }^{7}$ Miegielsen (2014) adopts a sequential search framework but considers a model where somehow consumers possess information about prices before engaging in search and the amount of information that consumers have (and share with each other) is given exogenously.
} 
wait until they receive an advertisement from the firm, or they are informed through their network. Instead, we allow consumers to actively reach out and search for information and we study markets where firms compete in prices. A more recent literature on networks concerns strategic interaction of competitors on networks. Chen et al. (2018) and Fainmesser and Galeotti (2020) study how firms price discriminate between different individuals depending on whether or not an individual influences many consumers. Campbell (2019) studies how in markets with product differentiation awareness of the available products is communicated through a social network and how this affects firms' prices and the efficiency of market outcomes. Campbell et al. (2020) consider how information about product quality of an experience good flows through the network and how this affects the quality provision by firms. None of these papers studies, however, the interaction between the incentives of consumers to acquire their own information through search and the diffusion of information through the social network and their impact on firms' prices and market outcomes.

The rest of the paper is organized as follows. The next section presents the baseline model, while Section 3 examines markets where consumers and firms are symmetrically (un)informed about the network structure. Section 4 provides the comparative statics analysis. Section 5 shows how the equilibrium outcome of the baseline model can be generated in a more general context. Section 6 continues the analysis by examining markets where consumers and firms are asymmetrically informed about the network: consumers know from how many friends they may acquire information before engaging in search activities, while firms only know aggregate network characteristics. We conclude with a discussion.

\section{The Baseline Model and Preliminary Results}

We consider a duopoly ${ }^{8}$ market for a homogeneous good where firms compete in prices. The unit cost of production is constant and normalized to zero. As firms may choose mixed strategies, we denote the strategy of a firm $i$ by $F_{i}(p)$, representing the probability

\footnotetext{
${ }^{8}$ With more than two firms, the characterization of the mixed strategy distribution in prices is more complicated and in this case, it is difficult to analyze the gains of search versus the gains of free riding. Galeotti (2010) also considers duopoly markets.
} 
that a firm charges a price not larger than $p$. The support of the price distribution is determined endogenously with $\underline{p}$ and $\bar{p}$ being the lower and upper bound of the support, with the possibility of some prices in the interior of the interval not being chosen.

On the demand side of the market, there is a countably infinite number of consumers, normalized to one, each with unit demand and a willingness to pay equal to $v>0$. A consumer buying at price $p$ receives a pay-off of $v-p$. If a consumer does not consume, she receives a payoff of zero. The consumers' choice situation is based on the sequential search paradigm (see, e.g., Kohn and Shavell (1974) and Stahl (1989)), where after obtaining one price quote at a search cost $s$, with $0<s<v$, consumers have to decide whether or not to continue to search. We add that because of WOM communication, consumers can also get information through their network of friends. This implies that we have to specify in detail how these two sources of information are intertwined. There are different ways to do this, but they all share the basic idea that at any point in the search process, consumers have the choice between actively searching themselves for price information from firms (which, like in the consumer search literature, involves a search cost), buying (which is only possible after being informed about at least one price), and waiting for friends to provide them with information (or not searching). The latter, we see as a period of time that the consumer is not active in the (online or offline) product market. In that period of time, one or multiple friends may have sent messages, and pay-offs come wih a time delay.

In this section we present a reduced form model with two periods to analyze this interaction. At the outset, in period 1, consumers individually and simultaneously decide whether to search or not. A consumer who decides not to search herself in period 1 cannot buy and has to wait until period 2. Searching consumers observe a price and can decide to immediately buy, or continue to search in the same period. The first search is random and costly and search is with perfect recall. Consumers who acquired information share this with their immediate neighbors in the social network. In the beginning of period 2 the non-searching consumers become active online, observe the information their friends have shared and decide whether to buy based on that information or to search themselves. Second-period pay-offs are discounted by a factor $0<\delta<1$.

The social network through which information is diffused is modeled by a given random graph. The probability a consumer has $k$ links is denoted by $t(k), k \in\{1,2, \ldots\}=O$ with 
$\sum_{k \in O} t(k)=1$. We denote by $q(k)$ the fraction of consumers with $k$ friends who choose to search themselves. In the next two sections, we first consider the case, however, where consumers do not or cannot condition their decision of becoming active searchers on the number of connections, and then $q$ is independent of $k$.

The timing of decisions is as follows. First, firms simultaneously set prices. Second, the network structure is realized (so that firms cannot condition their pricing strategy on the details of the network structure). Third, not knowing the prices, consumers simultaneously choose their strategies as described above.

A few comments on the interpretation of the model are in order. First, the reduced form interaction between search and word-of-mouth is arbitrary in many ways, e.g., in that it only allows for two periods, that a searching consumer cannot decide to not continue to search and wait for information from friends and that consumers only receive information from direct friends in the network so that information decays after one step. In Section 5 we show that provided that the search cost is small enough, the equilibrium outcome of this reduced form model is identical to the equilibrium outcome of a more general multiperiod model where in every period consumers may decide to buy using the information they have, search themselves, or not search (and go to the next period where they may receive more information from friends). We also discuss that our qualitative results are not affected if information flows to everyone who is connected. Second, we take the point of view that consumers engage in their social network for many reasons, not only to exchange price information through friends. Thus, the social network is given and we do not study the incentives to form links. Third, we think of $\delta$ as a measure of the speed of information communication. If $\delta$ is high, communication is fast and non-searching consumers quickly obtain information from searching friends. What is important is that there is some time elapse before a non-searching consumer becomes active (online) again and that within that period she may have obtained information from multiple friends. The advantage of receiving information via friends instead of actively searching oneself is to economize on search cost. A consumer can simply follow a referral and purchase without incurring a cost. ${ }^{9}$ Fourth, following the literature on observational learning (see,

\footnotetext{
${ }^{9}$ Alternatively, one could decompose the full cost of searching for and buying from a firm into two parts: a true cost of search and a cost of buying the good. In that case, following a referral consumers still have to incur the buying cost. In Atayev and Janssen (2019) we show that the analysis will be largely
} 
Kircher and Postlewaite (2008), Garcia and Shelegia (2018)), we also could have assumed that consumers can credibly exchange information only after having purchased goods. In Atayev and Janssen (2019) we show that our analysis applies equally to this case.

We use symmetric perfect Bayesian equilibria (PBE) as solution concept. A PBE is described by a set of firms' and consumers' strategies such that each is choosing optimally given beliefs and the strategies of the others.

In the remaining of this section, we show two preliminary results. First, any PBE where trade occurs is a so-called reservation price equilibrium, where consumers buy if, and only if, the price they observe is below a threshold price. Denote by $r$ the reservation price at which consumers are indifferent between buying immediately and continuing to search. As consumers' pay-offs of buying and continuing to search after observing a price $\widetilde{p}$ are given by $v-\widetilde{p}$ and $v-(1-F(\widetilde{p})) \widetilde{p}-F(\widetilde{p}) E(p \mid p<\widetilde{p})-s$, respectively, it is easy to see that $r$ is implicitly defined by

$$
F(r)(r-E[p \mid p \leq r])=s .
$$

If there is a solution to (1), it must be unique for any non-degenerate $F(p)$.

The following lemma rules out all equilibria where after receiving some information, consumers decide to continue to search. Thus, a searching consumer will always immediately buy if the price is smaller than $v$ and non-searching consumers who have received price information from friends will not decide to search themselves.

Lemma 1 Any symmetric equilibrium where goods are bought has $F(r)=1$.

This lemma is important in that it rules out equilibria where firms charge prices such that it is in the consumers' interest to continue to search. The result is familiar from the literature on sequential consumer search: if an equilibrium with prices $p$ larger than $r$ would exist, a firm's price will always be compared with another price, which incentivizes a firm to undercut the price of the competitor and not to set the largest price in the support of $F(p)$.

The second preliminary result is that there always exists a trivial "no trade" equilibrium.

unaffected as the buying cost effectively reduces the willingness to pay. 
Lemma 2 For any $s>0$, there exists an equilibrium without sales where $q(k)=0$ for all $k$.

As the first search is costly, this not surprising: knowing they will not sell to anyone, firms may set prices larger than $v-s$ and this pricing behavior rationalizes consumers' beliefs that it is not rational to search. If no one searches, no information is shared and consumers cannot buy.

\section{Active Markets}

In this section and the next, we focus on symmetric equilibria with positive sales, i.e., $0<q<1$. As $F(r)=1$, all searching consumers make a purchase at the first search so that a searching consumer's expected payoff is equal to

$$
v-E[p]-s
$$

Given that non-searching consumers will always follow the information they are provided with and will search in period 2 if they did not receive information from friends, their expected payoff is given by

$\delta \sum_{k \in O} t(k)\left[v-\sum_{m=1}^{k}\left(\begin{array}{c}k \\ m\end{array}\right) q^{m}(1-q)^{k-m}\left(\frac{1}{2^{m-1}} E[p]-\left(1-\frac{1}{2^{m-1}}\right) E_{\min }[p]\right)-(1-q)^{k}(E[p]+s)\right]$

This expression can be understood as follows. A non-searching consumer always makes a purchase either because she gets informed about a price from friends or she searches herself. The probability that $m$ of a consumer's $k$ friends search is equal to $\left(\begin{array}{c}k \\ m\end{array}\right) q^{m}(1-$ $q)^{k-m}$. They all visit the same firm with probability $1 / 2^{m-1}$, in which case she buys at the expected price. When the friends happen to search different firms, which happens with probability $1-1 / 2^{m-1}$, the consumer pays the lowest of the two prices, which in expected terms is $E_{\min }[p]$. The probability that none of a consumer's $k$ friends search is $(1-q)^{k}$, in which case the consumer searches herself incurring $s$ and buys from the first visited firm paying $E[p]$. The entire payoff depends on how fast information arrives to the consumer, and thus on the discount factor $\delta$.

To be able to handle these expressions, it is useful to introduce for any arbitrary 
$0 \leq x \leq 1$, the function $\tau(x)=\sum_{k \in O} t(k) x^{k}$, as the probability generating function. Two expressions will be of particular importance in our analysis: with if each consumer searching with probability $q, \tau(1-q)$ represents the probability that a consumer has only friends that do not search themselves, whereas $1-\tau\left(1-\frac{q}{2}\right)$ represents the conditional probability that a consumer who has obtained one price quote already obtains information concerning the competitor's price through the network of friends. It follows that

$$
\sum_{k=1}^{N} t(k) \sum_{j=0}^{k}\left(\begin{array}{l}
k \\
j
\end{array}\right) q^{j}(1-q)^{k-j} y^{j}=\tau(q y+(1-q)) .
$$

As $\tau(x)$ is a convex function with $\tau(1)=1$ and $1-\frac{q}{2}=(1-q+1) / 2$, it follows that $\widetilde{\tau}(q) \equiv$ $1+\tau(1-q)-2 \tau\left(1-\frac{q}{2}\right)>0$ for all $q>0 ; \widetilde{\tau}(q)$ is the ex ante probability that a consumer's friends have observed two different prices. Also, $\tau^{\prime}(1)=\lim _{q \rightarrow 1} \tau(1-q) /(1-q)=t(1)$.

The probability $q$ is determined such that the consumer is indifferent between searching and not searching and, therefore, in equilibrium the above two expressions have to be equal. Using the probability generating function, the indifference condition can be written as

$$
(1-\delta)(v-E[p])=(1-\delta \tau(1-q)) s+\delta \widetilde{\tau}(q)\left(E[p]-E_{\min }[p]\right)
$$

We now turn to the determination of the equilibrium pricing strategy of the firms. Setting price $p$, an individual firm's expected profit is given by

$$
\Pi(p)=\left(\frac{q}{2}+\delta(1-q)\left\{\sum_{k \in O} t(k) \sum_{m=1}^{k}\left(\begin{array}{l}
k \\
m
\end{array}\right) q^{m}(1-q)^{k-m}\left[\frac{1}{2^{m}}+\left(1-\frac{1}{2^{m-1}}\right)(1-F(p))\right]\right\}+\frac{(1-q)^{k}}{2}\right) p
$$

Clearly, a consumer is active with probability $q$ and half of the times she visits the firm under question to buy outright. With probability $1-q$, a consumer is passive in which case she purchases only after the information arrives to her, thus speed of information diffusion $\delta$. She definitely buys from the firm if all of her $m$ (out of $k$ ) active friends happen to visit the firm, which happens with probability $1 / 2^{m}$. With probability $1-1 / 2^{m-1}$, these active friends happen to visit different firms, in which case the firm under question makes sales only if its price is lower than that of the rival firm, or $1-F(p)$. Finally, with probability $(1-q)^{k}$ none of her friends search, in which case a consumer searches herself and buys from the firm half of the time. Using the probability generating functions, the profit 
expression can be rewritten as follows: $E_{\min }[p]$

$$
\Pi(p)=\left(\frac{q}{2}+\delta(1-q)\left[\tau\left(1-\frac{q}{2}\right)-\frac{\tau(1-q)}{2}+\widetilde{\tau}(q)(1-F(p))\right]\right) p
$$

Equating these expected profits with the profit of setting a price equal to the upper bound of the distribution gives the equilibrium price distribution as

$$
F(p)=1+\eta-\eta \frac{\bar{p}}{p}, \text { with support }[\underline{p}, \bar{p}]
$$

where

$$
\eta=\frac{\frac{q}{2}+\delta(1-q)\left(\tau\left(1-\frac{q}{2}\right)-\frac{\tau(1-q)}{2}\right)}{\delta(1-q) \widetilde{\tau}(q)}>0,
$$

and $\underline{p}=\frac{\eta}{1+\eta} \bar{p}$ solves $F(\underline{p})=0$, whereas $\bar{p}=\min \{r, v\}$. The fraction $\eta$ is the ratio of consumers who do not compare prices to those that do compare prices (as in the traditional models of Varian (1980) and Stahl (1989)). Here, the fraction of consumers who are informed about only one price consists of the fraction of searching consumers and those non-searching consumers who receive only one price quotation from friends, while the fraction of consumers who are informed about both prices only consists of non-searching consumers who receive through their social network information about the prices of both firms.

Let us first discuss the extreme cases where $\delta=0$ or $\delta=1$. In these cases, the Diamond paradox emerges and only an equilibrium with no trade exists. If $\delta=0$, there is no advantage to not search and no consumer ever compares prices. The left-hand side of (2) reduces to $v-E[p]$ and the right-hand side to $s$. Consumers choose to be active if the expected price is below $v-s$ and drop out of the market if it is greater than $v-s$. From (4) it is clear that $\eta$ (and thus expected price) become infinitely large. Hence, consumers prefer not to search. On the other hand, if $\delta=1$, there is no advantage to search so that $q=0$. If no consumer acquires information herself, no one compares prices and again $\eta$ (and thus expected price) become infinitely large. In this case, the ex ante probability that a consumer's friends have observed two different prices $\widetilde{\tau}(0)=0$.

Proposition 1 If $\delta=0$ or $\delta=1$, there does not exist an equilibrium with active trade.

Having explained the different conditions that should hold in an RPE with positive 
sales, we are now able to provide the main result of this section, namely that for any $0<\delta<1$ an equilibrium exists if the search cost is sufficiently small.

Theorem 1 For any given $0 \leq t(1)<1$ and $0<\delta<1$, there exists a $\bar{s} \leq v$ such that an RPE exists if, and only if, $s \leq \bar{s}$. If an RPE exists it is determined by the triple $(q, r, F(p))$ solving (1), (2), and (3). Furthermore, as $s \rightarrow 0$ the optimal search probability $q$ converges to 1 , while price dispersion disappears with $\bar{p}=\underline{p}=v$.

The proof of the proposition is given in the appendix. The main intuition can be understood as follows. If a fraction $0<q<1$ of consumers actively search themselves, while the others are passive, then there is a strictly positive probability that some of the inactive consumers are informed about two prices so that the price distribution of firms is non-degenerate. The main challenge then is to find a value of $q$ such that consumers are indifferent between searching and non-searching. The proof shows that when $s \leq \bar{s}$ there exists such a $q$. It is clear that $\bar{s}$ depends on the exogenous parameters and, in particular, in line with Proposition 1 , that $\bar{s}$ approaches 0 as $\delta$ approaches 0 or 1 .

The interesting aspect is that if a fraction $0<q<1$ of consumers search, while the others do not, then as long as some consumers have more than one connection, i.e., $t(1)<1$, it is the fraction of the non-searching consumers who are free-riding that provide a positive service to the searching consumers as they are the ones who comapre oth prices. Thus, the non-searching consumers play a crucial role to resolve the Diamond paradox.

Importantly, if $s$ becomes arbitrarily small, price dispersion must vanish as consumers can obtain another price quote at virtually no additional cost. If $s=0$, any non-negative price $p^{*} \leq v$ can be sustained in equilibrium. Consumers' strategy is to search $(q=1)$ and $r=p^{*}$ : they buy immedietaly if they observe a price $p \leq p^{*}$, otherwise they continue to search. If $s \rightarrow 0$ the model selects the equilibrium where firms price at the monopoly level. Given that price dispersion disappears, the share of non-searching consumers must disappear as non-searching consumers pay almost the same price as searching consumers, but incur a cost of waiting as $0<\delta<1$. For any $s>0$ firms have the market power to raise their price up to the consumers' reservation price, which is larger than the expected price. As in the Diamond paradox, this will raise the expected price until the monopoly price $v$. Interestingly, and different from the Diamond paradox, consumers choose to be search as for any $s>0$ there remains some price dispersion and the expcted price is 
smaller than $v$.

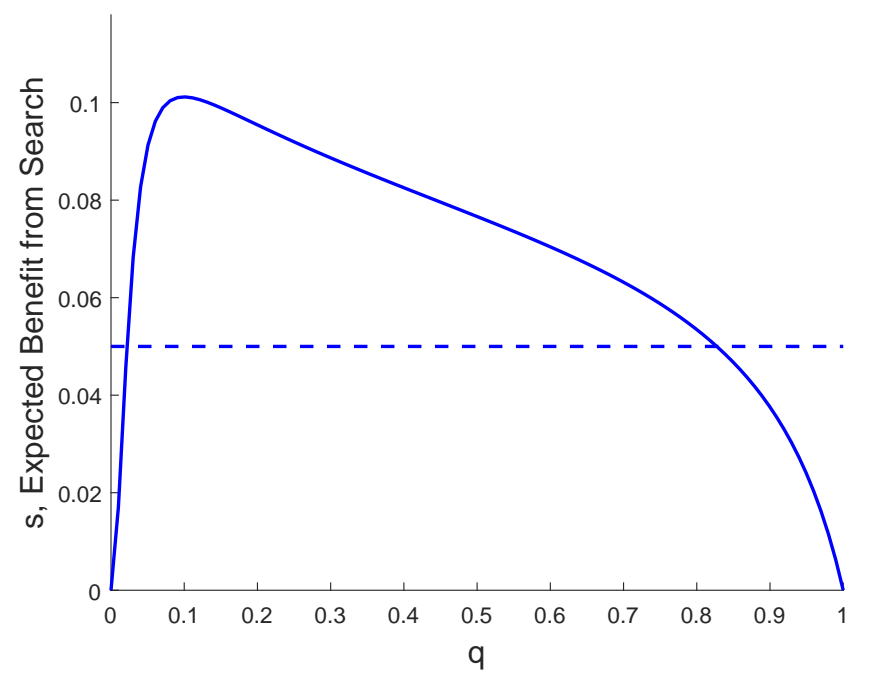

Figure 1: An illustration of existence of an RPE for $\delta=0.9, s=0.05$ and $t(k)=n k^{\gamma}$, where $\gamma=-1, \bar{k}=100$ and $\sum_{k \in O} n k^{\gamma}=1$.

Figure 1 illustrates the equilibrium construction. The horizontal axis represents the fraction $q$ of searching consumers, while the cost and the expected benefit of search are presented on the vertical axis. The solid curve represents the expected benefits, while the dashed horizontal line represents the cost of search. In the proof we show what the figure presents, namely that when $q$ approaches 0 or 1 the expected benefit of search approaches 0 . This is quite intuitive: if $q$ approaches 0 or 1 , there are very few consumers who compare prices either because there are almost no non-searching consumers $(q$ close to 1 ) or because there is almost no information that is diffused in the system ( $q$ close to $0)$. More formally, this can be seen as $\eta \rightarrow \infty$ if $q$ approaches 0 or 1 . Thus, firms exercise their market power, and prices get close to $v$. As for interior values of $q$ the expected benefits are positive and continuous in $q$, it must be the case that for small enough values of $s$ an equilibrium exists.

The figure shows that for (given) small enough values of $s$ there are two intersection points and, hence, two equilibrium values of $q$ where the market is active. ${ }^{10}$ One may argue, however, as in other search models (see, e.g. Burdett and Judd (1983), Fershtman and Fishman (1992), Janssen and Moraga-Gonzalez (2004) and Honda (2015))

\footnotetext{
${ }^{10}$ For certain parameter configurations with high search costs, there may exist four equilibria with positive trade. For our comparative static analysis, we focus on sufficiently small search costs such that only two equilibria with active trade exist.
} 
that the equilibrium corresponding to the higher search probability can be called a "stable" equilibrium in the sense that if the real search probability falls (slightly) short of the equilibrium value the expected benefit of search exceeds the cost so that consumers have an incentive to search more intensively. It follows that if the search cost asymptotically approaches zero, the optimal search probability in the active equilibrium approaches $1 .^{11}$ The comparative static analysis focuses on this "stable" equilibrium.

\section{Comparative Statics}

Given the equilibrium characterization, we now can provide insights into how market outcomes depend on exogenous parameters. We will first focus on the impact of network structure on equilibrium prices, before concentrating on the speed of communication in the network (represented by $\delta$ ), and the impact of the cost of searching $s$. A social network like Facebook has significantly increased the number of connections people have (although there remain a non-negligible fraction of consumers who do not use Facebook or other social networks) and the speed of information diffusion through the network. Online markets have significantly reduced search cost $s$. In this section, we discuss the implications of these effects on market outcomes, especially the expected market price. Unless explicitly discussed otherwise, the changes in firms' profits is perfectly in line with expected price (as in an RPE eventually all consumers buy). In an RPE, the expected market price is proportional to $s$ and given by $E[p]=s\left(\frac{\eta \ln \left(1+\frac{1}{\eta}\right)}{1-\eta \ln \left(1+\frac{1}{\eta}\right)}\right)$, which is increasing in $\eta$.

We investigate the limiting behavior when the network of consumers is dense and all consumers tend to have many links. One may think that if all consumers potentially get information from many friends, competition would prevail and prices converge to marginal cost with price dispersion being eliminated. Surprisingly, however, as the next result shows, price dispersion remains an essential feature of any reservation price equilibrium, and even in the limit when all consumers have infinitely many links, price dispersion remains bounded away from 0 .

\footnotetext{
${ }^{11}$ The figure also shows there is a stable equilibrium where the market is inactive $(q=0)$ and $(2)$ does not hold.
} 
Proposition 2 For any $s>0$, any RPE with active search is characterized by price dispersion even if all consumers tend to have infinitely many links.

A consequence of the limiting price dispersion is that prices do not converge to marginal cost. The reason that price dispersion remains is that in any RPE without price dispersion, the reservation price, and hence, the expected price should be infinitely large. But then consumers are better off not searching.

It is important to understand that for this result to hold the first search should be costly. If this were not the case, all consumers would search and the probability of obtaining the second price from friends then approaches one as the number of links to each consumer in the population becomes large. As a consequence, market frictions would disappear and prices converge to marginal cost. This also explains why in Galeotti (2010) prices do converge to marginal cost if the network of consumers gets dense: it is the consequence of the first search being assumed to be free in his model.

By means of numerical simulations, we can analyze intermediate cases of network connectivity. We assume the social network can be described by a random graph that follows a power law ${ }^{12} t(k)=n k^{\gamma}$, where larger $\gamma$ values stand for denser networks with consumers having more connections. In particular, comparing two networks with probabilities of connections being given by $t(k)$ and $\widetilde{t}(k)$, the network with probabilities $t(k)$ is generated by a higher $\gamma$ (and thus, denser) if there exists a $\widetilde{k} \in O$ such that $t(k)<\widetilde{t}(k)$ for all $k<\widetilde{k}$, whereas $t(k)>\widetilde{t}(k)$ for all $k>\widetilde{k}$. In Figures 2 and $3,{ }^{13}$ we gradually increase $\gamma$ from -2 to 2 .

Figure 3 depicts the impact of network density on the expected price. It shows that the expected price is decreasing in $\gamma$ and that certainly when the network is not very dense ( $\gamma$ is small), this impact is quite strong as the expected price may decrease by around $50 \%$ as $\gamma$ increases from around -2 to 0 . The main, direct impact can be understood by noting that if the network is described by the power law $t(k)=n k^{\gamma}$ it follows from (4)

\footnotetext{
${ }^{12}$ Empirical analysis has demonstrated that many social networks can be described by a power-law of the form we assume here (see, for example, Price (1965)).

${ }^{13}$ The following parameter values have been used: $v=1, s=0.05, \delta=0.9$, and $\bar{k}=100$.
} 
that the ratio $\eta$ is given by

$$
\eta=\frac{\frac{q}{2}+\delta(1-q) \sum_{k \in O} n k^{\gamma}\left[\left(1-\frac{q}{2}\right)^{k}-\frac{(1-q)^{k}}{2}\right]}{\delta(1-q)\left(1+\sum_{k \in O} n k^{\gamma}\left[(1-q)^{k}-2\left(1-\frac{q}{2}\right)^{k}\right]\right)} .
$$

As the term in square brackets in the numerator is positive and decreasing in $k$, while the term in square brackets in the denominator is negative and increasing in $k$, it follows that networks with higher $\gamma$ 's put relatively more weight on a higher number of connections, which implies that $\eta$ is decreasing in $\gamma$. As lower $\eta$ 's reflect the fact that there are relatively more price comparing consumers in the market, the direct effect puts downward pressure on prices. There is, however, also an indirect effect through the consumers' search probability $q$. A decrease in price levels is associated with a decrease in price dispersion, as measured by the difference $E[p]-E_{\min }[p]$. This makes it more attractive for consumers to search as the main benefit of not searching, namely being informed about both prices and therefore being able to buy at the lowest of the prices, becomes smaller. In addition, as the expected price is lower, consumers would like to have the larger benefit of a purchase now rather than having to wait for information through friends. The associated increase in the share of searching consumers as illustrated in Figure 2 increases $\eta$ and therefore also increases expected price. This indirect effect is, however, smaller than the direct effect and thus, the overall impact on expected price is decreasing.

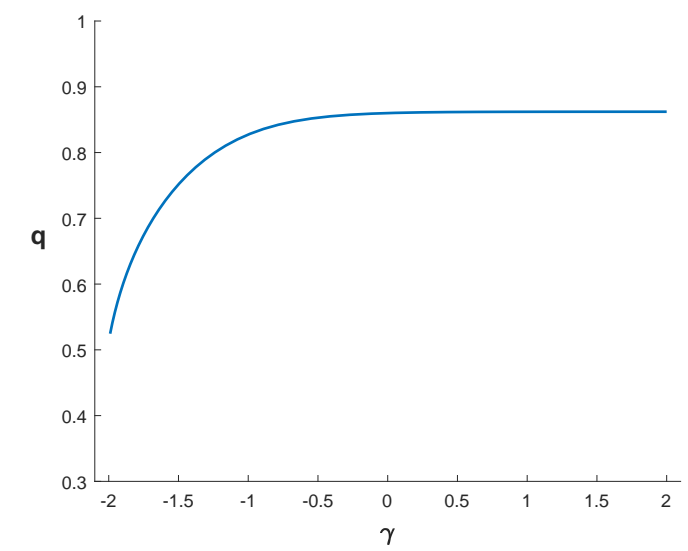

Figure 2: Impact of network density on the share of active consumers

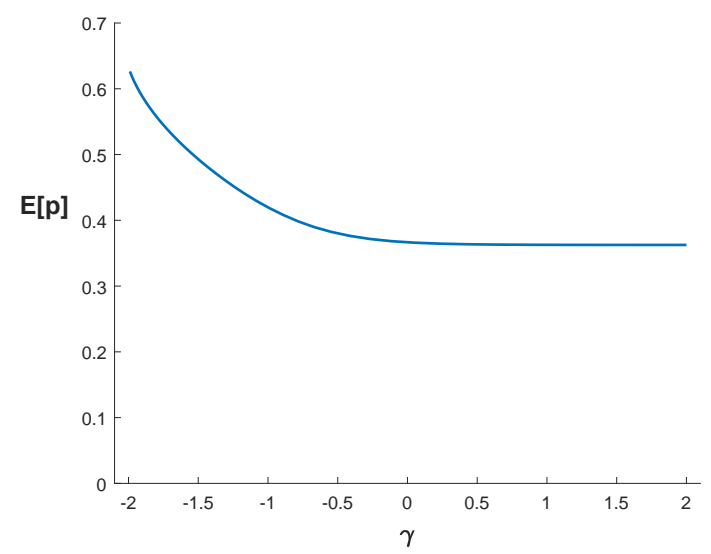

Figure 3: Impact of network density on expected price

Note that the above result on the fraction of consumers actively acquiring information 
is strikingly different from Galeotti (2010) and Galeotti and Goyal (2010) where an agent's probability of actively acquiring information negatively correlates with the number of links she has. Intuitively, one might expect that the more connections a consumer has the less likely she is to become active as, all else being equal, the probability that she obtains information from friends rises. In our case, however, it is the combined price effect through a lower expected price and lower price dispersion as measured by $E[p]-E_{\min }[p]$ that overrides this intuitive effect.

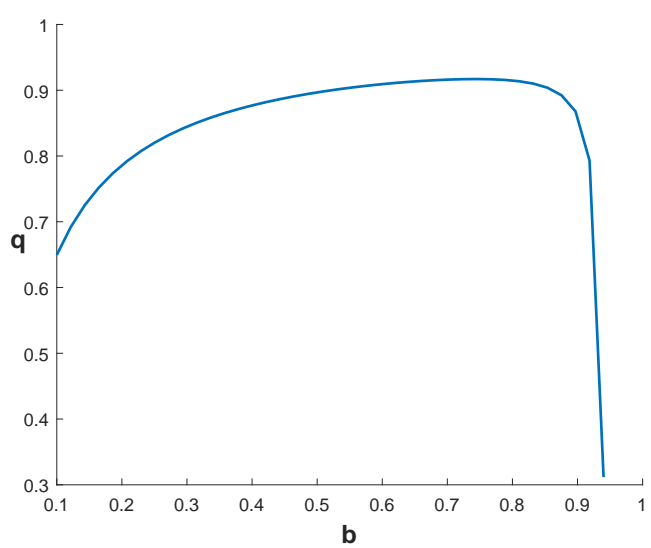

Figure 4: Impact of $\delta$ on the share of active consumers

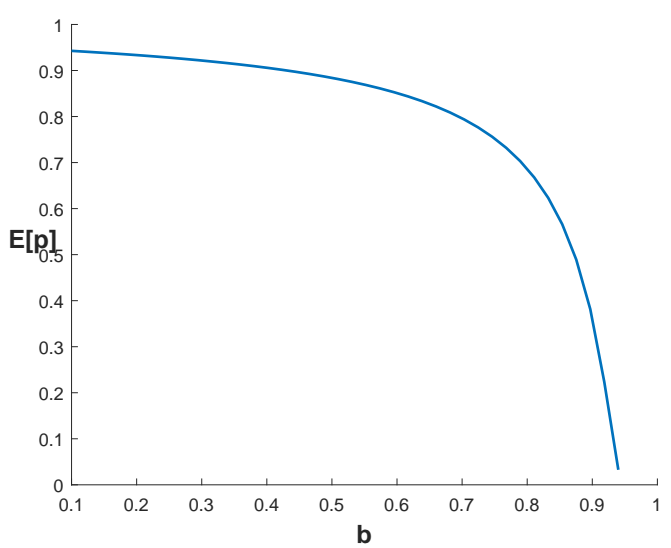

Figure 5: Impact of $\delta$ on expected price

Next, we investigate the impact of a change in $\delta$. Social media have significantly increased the speed with which consumers may share information, which in our model is measured by $\delta$. We know that a higher $\delta$, resulting in faster information transmission, permits a consumer to access information quickly from friends, but that this may have repercussions on the incentives to search. Proposition 3 establishes that at extreme values of $\delta$ an equilibrium with positive trade does not exist. Numerical simulations in Figures 4 and 5 show the effect of an increase in $\delta$ on the share of searching consumers and the expected price when we change the value of $\delta$ in an intermediate range (here from 0.1 to 0.94$)$. For larger values of $\delta$, an equilibrium with search ceases to exist. ${ }^{14}$ As before, there is a direct and an indirect effect of $\delta$ on prices. The direct effect can be seen by taking the derivative of $\eta$ with respect to $\delta$ in (4). It is easy to see that this derivative is negative so that an increase in the speed of information processing in the population increases the share of price comparing consumers. This effect reduces prices. There is also

\footnotetext{
${ }^{14}$ The Figures are drawn for the following parameter values: $v=1, s=0.05, t(k)=n k^{\gamma}, \bar{k}=100$, and $\gamma=0$.
} 
an indirect effect via $q$, however. For small values of $\delta$ the indirect effect is very similar to the indirect effect we mentioned in relation to the impact of $\gamma$, namely that as prices and price dispersion decline, consumers have more incentives to become active searchers themselves. In this case, there is, however, also a direct effect of $\delta$ on $q$ (as shown in Equation (2)). As $\delta$ becomes large, there is almost no downside to waiting anymore and consumers massively change their behavior and do not search. As, focusing on stable equilibria, $\eta$ is increasing in $q$ this further strengthens the direct effect so that the total effect of $\delta$ on the expected price becomes more pronounced.

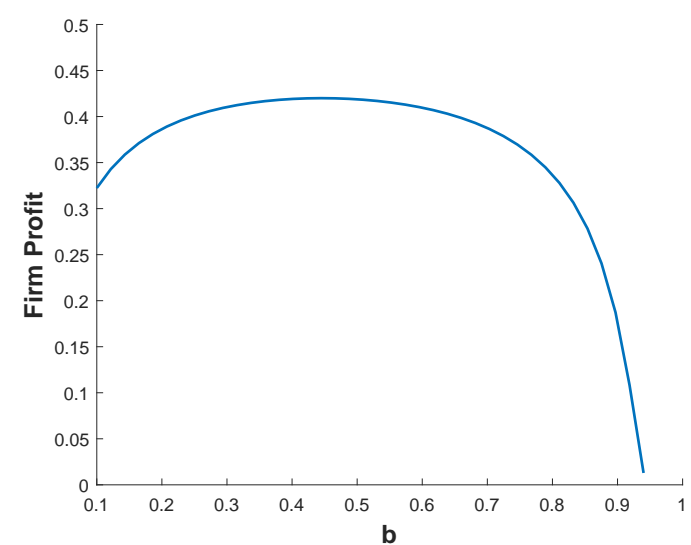

Figure 6: Impact of $\delta$ on firm profit

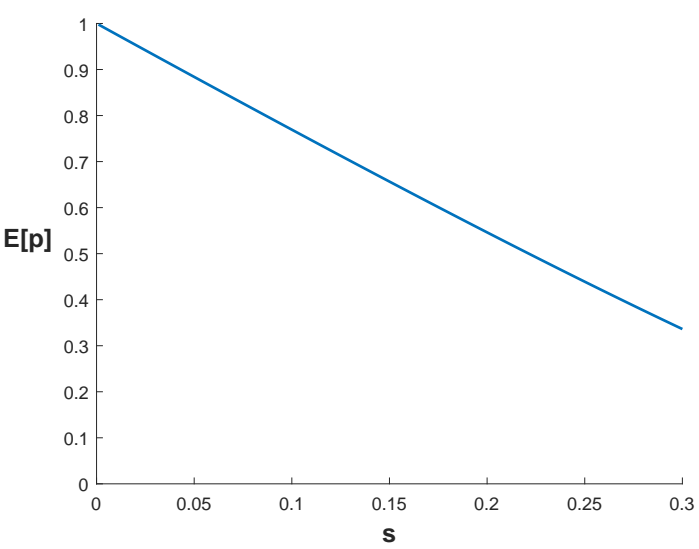

Figure 7: Impact of $s$ on expected price

Interestingly, the speed of information diffusion also has a non-monotonic effect on firms' profits as shown in Figure 6. When the speed of information diffusion is relatively low, firms have an incentive to increase the number of searching consumers as these consumers buy immediately, whereas the sales to non-searching consumers are heavily discounted. Thus, even though expected price is monotonically decreasing in the speed of information diffusion, the fact that the fraction of searching consumers is increasing offsets the decrease in revenue per consumer. On the other hand, when the speed of information diffusion is already relatively high to begin with, firms would not want to increase it further. At some point, if the speed of information diffusion is very large, both firms and consumers suffer from a further increase in the diffusion speed as an equilibrium with active trade ceases to exist.

Finally, we proceed by studying the impact of a change of $s$. We already know that the optimal search probability goes to 1 in the limit when $s$ goes to zero, suggesting that as $s$ starts increasing from 0 the optimal search probability decreases. The next proposition 
shows that the underlying effects also hold true outside the region where $s$ is close to 0 .

Proposition 3 In any stable RPE, the share of active consumers is decreasing in $s$.

We know from Theorem 1 that in the limit when $s$ is arbitrarily small, prices converge to the monopoly price $v$. This immediately implies that for small enough search cost, the expected price must be decreasing in $s$ as more consumers make price comparisons and firms will compete for these consumers. As $\eta$ is increasing in $q$ and expected price is increasing in $\eta$, it follows that the expected price is decreasing in $s$ for larger values of $s$ as well, as illustrated in Figure $7 . .^{15}$

\section{A Foundation for the Baseline Model}

There are many ways to model the interaction between sequential search and word-ofmouth communication. The baseline model we have analyzed so far is simplistic as $(i)$ it only allows for two periods and ( $i i)$ a searching consumer cannot decide to not continue to search and wait for information from friends. In this section we show that for small search costs the main results of the previous sections continue to hold in a more general multiperiod model where in every period consumers may decide to buy using the information they have, search themselves, or not search (and go to the next period where they may receive more information from friends) provided that $t(1)>0$.

We also show that the formal equivalence between the equilibrium outcome of the baseline model and the more general model depends on consumers only sharing information they have acquired themselves. This is similar to the setting studied in Bramoulle and Kranton (2007), Banerji and Dutta (2009) and Ellison and Fudenberg (1995) where information decays after one link in the network. The qualitative results continue to hold, however, if information flows to all directly or indirectly connected consumers.

So, let us first analyze the general model where at every decision moment consumers can choose to search themselves, buy from one of the firms from which they have obtained information or not search with the following three conditions: $(i)$ a consumer can only buy if she is informed of at least one price, $(i i)$ if a consumer decides to not search, then

\footnotetext{
${ }^{15}$ We use the following parameter values for the figure: $v=1, t(k)=n k^{\gamma}, \bar{k}=100, \gamma=0, \delta=0.5$.
} 
she "waits" and she is moved to the next period where her pay-offs are discounted by $\delta$, and (iii) information exchange takes place at the beginning of every period. Thus, the following dynamic unfolds. After firms have chosen their prices and the network is formed, consumers at the beginning of their first "search period" can choose to search or not search. Not searching consumers have to wait for taking actions until the next period. After their first search, searching consumers can decide to buy, continue to search (and then decide where to buy) or not search anymore in this round. Then, the second "search period" starts and information is exchanged: everyone that acquired information shares with her immediate friends, and all consumers that did not buy yet can choose to search, buy or not search subject to the above three conditions. This process continues as long as some consumers have not bought yet and still consider buying at some point.

We now show by means of a series of lemmas that in this general model, $(i)$ all consumers who decided not to search in the first "search period" and who did not receive any price information from friends at the beginning of the second "search period" will search themselves and ( $i i)$ all consumers who have acquired price information either from friends or through their own search activity will buy immediately, i.e., they will not continue to search and they will not decide not to search and wait until the next period. It then follows that the equilibrium outcome is exactly the same as in the baseline model so that the fraction $q$ of searching consumers in the first "search period" is determined by the same indifference condition (2) and also $\eta$ is determined by (4).

First, as for any $0<q<1$ some consumers will be informed about both prices, there cannot be mass points in the symmetric price distribution as it will pay for firms to undercut. But then a firm that charges an upper bound $\bar{p}>r$ will not sell to any consumer.

Lemma $3 F(p)$ does not have atoms and $F(r)=1$.

Next, we show that for $s$ close enough to 0 , it should be that $q$ is close to 1 . As the reservation price $r$ continues to be defined by 1 , it follows that for $s$ close to $0 r \approx E[p]$, but given that there are no mass points, it then also follows that $E[p] \approx E\left[\min \left\{p_{1}, p_{2}\right\}\right]$. As price dispersion only disappears for $q$ close to 0 or 1 , the stable equilibrium is the one where $q$ is close to 1 .

Lemma 4 If $s$ is close enough to 0 , then $q$ is close to 1. 
Another point in the overall argument is that consumers who did not search in the first "search period" want to search in the second "search period" if they did not get information from friends. The argument essentially is that it is much more likely to be informed by friends in the beginning of the second period than it is in the beginning of the third period. What is important in this regard is that the probability that a consumer will be informed by a friend at the beginning of the second period is given by $\sum t(k)\left[1-(1-q)^{k}\right]$, while the probability $x$ that a consumer's friend will be informed by their friends, knowing that I did not search, equals $x=\sum t(k)\left[1-(1-q)^{k-1}\right]$. This latter probability is important as, in case information decays after one link in the network, a consumer cannot count on receiving information from friends in future periods if they bought the product after receiving information from their friends that they do not pass on. The probability that I will be informed by my friends in the third period after I did not receive information from them in the second period is then maximally equal to $\sum t(k)(1-x)^{k}$ as at that moment I can only be informed by friends who searched themselves in the second period (and who thus did not get information from their friends). For $s$ close to 0 and $q$ close to $1, x \approx 1-t(1)$ so that $\sum t(k)(1-x)^{k} \approx \sum t(k) t(1)^{k}<$ $t(1)<1$. Thus,

Lemma 5 If $s$ is close enough to 0, then consumers who did not search from the start want to search after they did not get information from friends.

It remains to be shown that if a consumer is informed about any price $p<r$, she does not want to wait for the next period. To this end, denote by $\rho$ the smallest price at which a consumer who is informed about that price would be indifferent between not searching (and wait until the next period) and buying. An active consumer who waits after searching the first firm receives a price quote of the other firm from friends with probability $1-\tau\left(1-\frac{q}{2}\right)$. Thus, we can write the payoff of an active buyer who observes price $\widetilde{p}$ and waits as $\delta\left[v-\left(1-\tau\left(1-\frac{q}{2}\right)\right) F(\widetilde{p}) E[p \mid p<\widetilde{p}]-\left[1-\left(1-\tau\left(1-\frac{q}{2}\right)\right) F(\widetilde{p})\right] \tilde{p}\right]$. Then, since the payoff from buying at price $\widetilde{p}$ is $v-\widetilde{p}, \rho$ is implicitly given by

$$
(1-\delta)(v-\rho)=\delta\left(1-\tau\left(1-\frac{q}{2}\right)\right) F(\rho)(\rho-E[p \mid p<\rho])
$$

The proof that a consumer does not want to wait if she is informed about a price 
$p<r$, is in two parts. First, in the Appendix we show that the RHS of (5) is increasing in $\rho$ so that

Lemma 6 If there is some $\rho<r$, it must be unique.

The last part of the argument builds on the fact that equilibria with active markets only exist if at least some consumers are searching and for this it must be true that consumers, who are ex ante identical, at least weakly prefer to search than not to search. We show that this weak preference implies that consumers would strictly prefer to continue to search than not to search after having observed a first price quote. The only reason for a searching consumer to wait after having observed a first price quote is that she hopes to be able to economize on the search cost $s$ by getting informed about the other price quote via a friend. However, the chance of being informed through the social network about the second price that is not yet observed is smaller than the chance of being informed about any price. Thus, if a consumer weakly prefers to search before observing a price, she strictly prefers to search than to wait after observing a price.

Lemma 7 In any equilibrium where there is active search, we have that $r \leq \rho$.

Together with $F(r)=1$ it follows that in any equilibrium with trade consumers immediately buy after observing a price. Thus, we have the following result:

Proposition 4 If $s$ is small enough and information decays after one link in the network, then all equilibria of the general model coincide with the equilibria of the baseline model.

\subsection{Information spreading through the network}

So far in this section we have continued to assume that consumers can only spread information if they have searched for it themselves, i.e., information decays after one link. In this subsection we consider an alternative case where information does not decay. The most straightforward way for information not to decay is that at the beginning of round 2 all information is spread to everyone who has a direct or indirect link to each other. ${ }^{16}$

\footnotetext{
${ }^{16}$ Alternatively, one could think that every period information is spread to direct friends only so that it takes two periods to obtain information that friends of friends acquired, and so on. As this is somewhat more cumbersome to analyze, we restrict ourselves to all information becoming available to everyone immediately.
} 
It is known that as the number of agents in a network grows without bounds, the network becomes fully connected if the probability that two random consumers are linked is larger than some cutoff probability. For example, in networks where the distribution of links over some $n$ number of agents is Poisson distributed, this cutoff probability is $\ln (n) / n$ (Theorem 4.1. in Jackson (2008)). Thus, the network becomes almost surely connected as $n \rightarrow \infty$ if the probability that a buyer has a link is greater than $\ln (n) / n$. In this section we assume that the network structure of our model satisfies the above condition.

It is clear that $F(r)=1$ should hold again. If all information is spread instantaneously to all consumers that are directly or indirectly linked to each other, a non-searching consumer can buy almost surely at the lowest of both prices so that the indifference condition determining $q$ is equal to

$$
v-E[p]-s=\delta\left(v-E_{\min }[p]\right),
$$

while firms' profit is given by

$$
\Pi(p)=\left(\frac{q}{2}+\delta(1-q)(1-F(p))\right) p .
$$

It follows that

$$
F(p)=1-\frac{q}{2 \delta(1-q)} \frac{r-p}{p} .
$$

Thus, we have a similar result as in Theorem 1:

Proposition 5 For any $0<\delta<1$, there exists a $\bar{s} \leq v$ such that an RPE exists if, and only if, $s \leq \bar{s}$. If an RPE exists it is determined by the triple $(q, r, F(p))$ solving (1), (6) and (7). Furthermore, as $s \rightarrow 0$ the optimal search probability $q$ converges to 1 , while price dispersion disappears with $\bar{p}=\underline{p}=v$.

\section{Information Asymmetry about Connections}

If consumers know the number of links they have before engaging in search, not all consumers search with the same probability. There may be consumers whose optimal search probability is between zero and one, and there will be ones who either definitely search or do not search at all. As consumers with more connections are more likely to obtain 
information via their social network than consumers with fewer links, the expected payoff from waiting is increasing in the number of connections. Thus, if a consumer with, say, $\widehat{k}$ connections is indifferent between becoming an active searcher and staying passive, so that $0<q(\widehat{k})<1$, then all consumers with more connections wait to obtain information through their social network, while those with fewer connections definitely search. If there is no consumer who is indifferent, then we can set $\widehat{k}$ to be the largest number such that $q(\widehat{k})=1$. Clearly, for any $s>0$ it cannot be the case that $\widehat{k}=\bar{k}$ and $q(\bar{k})=1$ as then all consumers would search themselves and no one would compare prices.

Proposition 6 Let $\widehat{k}, 1 \leq \widehat{k} \leq \bar{k}$ be as defined above. Consumers with a number of friends less than $\widehat{k}$ search with probability one and consumers with more than $\widehat{k}$ friends do not search.

Thus, consumers with more connections are more inclined to not search themselves and wait for information from their friends. It is clear that from a social welfare perspective, the wrong set of people search. Keeping the fraction of active consumers constant, if people with more links would search instead, there are two effects. First, the welfare loss of passive consumers who do not acquire any information and still need to search themselves is smaller as their fraction will be smaller. Second, as more consumers would make price comparisons, there is a redistribution of surplus from firms to consumers.

Given this result, the consumer behavior in any equilibrium can be characterized by three parameters; $\widehat{k}, q(\widehat{k})$ and a reservation price $r$. In what follows we use $q$ as a shorthand notation for $q(\widehat{k})$.

Correctly anticipating the optimal behavior of consumers, an individual firm's ex- 
pected profit of setting a price equal to $p \leq r$ is equal to

$$
\begin{aligned}
\Pi(p)= & \frac{1}{2} \widehat{w} p+\delta t(\widehat{k})(1-q)\left[\sum_{m=1}^{\widehat{k}}\left(\begin{array}{c}
\widehat{k} \\
m
\end{array}\right) w^{m}(1-w)^{\widehat{k}-m}\left(\frac{1}{2^{m}}\right)+\frac{(1-w)^{\widehat{k}}}{2}\right] p \\
& +\delta t(\widehat{k})(1-q) \sum_{m=1}^{\widehat{k}}\left(\begin{array}{c}
\widehat{k} \\
m
\end{array}\right) w^{m}(1-w)^{\widehat{k}-m}\left(1-\frac{1}{2^{m-1}}\right)(1-F(p)) p \\
& +\delta\left[\sum_{k=\widehat{k}+1}^{\bar{k}} t(k) \sum_{m=1}^{k}\left(\begin{array}{c}
k \\
m
\end{array}\right) w^{m}(1-w)^{k-m}\left(\frac{1}{2^{m}}\right)+\frac{(1-w)^{\widehat{k}}}{2}\right] p \\
& +\delta \sum_{k=\widehat{k}+1}^{\bar{k}} t(k) \sum_{m=1}^{k}\left(\begin{array}{c}
k \\
m
\end{array}\right) w^{m}(1-w)^{k-m}\left(1-\frac{1}{2^{m-1}}\right)(1-F(p)) p,
\end{aligned}
$$

where $\widehat{w}=\sum_{k=1}^{\widehat{k}-1} t(k)+t(\widehat{k}) q$ is the average search probability of a consumer and $w=$ $\frac{\sum_{k=1}^{\widehat{k}-1} t(k) k+t(\widehat{k}) \widehat{k} q}{\sum_{k=1}^{\bar{k}} t(k) k}$ is approximately the search probability of a consumer's neighbor (not knowing how many friends the neighbor has; cf., Section 4.2. in Jackson (2008)).

This expression can be understood as follows. A fraction of $\sum_{k=1}^{\widehat{k}-1} t(k)$ consumers has less than $\widehat{k}$ links. They search themselves and visit the firm with probability 0.5 . Since they do not compare prices they pay the price charged by the firm (if this is not larger than their reservation price). Consumers with $\widehat{k}$ links, who make a share of $t(\widehat{k})$ of the population, search with probability $q$ and visit the firm in half of the cases, and these consumers along with those with fewer links give the first term in (8). With probability $(1-q)$, consumers with $\widehat{k}$ links do not search and can be informed by their friends about price(s). Each of their friends searches with an expected probability $w$. Then, consumers obtain information only about the firm's price if all of their searching friends, represented by $m$, happen to visit that firm, which occurs with probability $\frac{1}{2^{m}}\left(\begin{array}{c}\widehat{k} \\ m\end{array}\right) w^{m}(1-w)^{\widehat{k}-m}$. If none of her friends search, meaning $m=0$, the consumer searches herself later and visits the firm half of the time. This gives the second term. The third term represents the probability that a consumer does not search and obtains information about both prices from her $m$ searching friends. The probability of this event is equal to $\left(1-\frac{1}{2^{m-1}}\right)\left(\begin{array}{l}\widehat{k} \\ m\end{array}\right) w^{m}(1-w)^{\widehat{k}-m}$. In this case, a consumer buys from the firm if the other firm charges a higher price than $p$, which happens with probability $1-F(p)$. Finally, the last two terms in the profit function account for the share of the population with more than $\widehat{k}$ links. These expressions are similar to the ones for the consumers with $\widehat{k}$ links who do not search themselves. As all 
waiting consumers buy with a delay (after the information about prices arrives to them), the payoff from these consumers is discounted by $\delta$.

The expected profit in (8) can be simplified as ${ }^{17}$

$$
\begin{aligned}
\Pi(p)= & {\left[\frac{\widehat{w}}{2}+\delta t(\widehat{k})(1-q)\left(\left(1-\frac{w}{2}\right)^{\widehat{k}}-\frac{(1-w)^{\widehat{k}}}{2}\right)+\delta \sum_{k=\widehat{k}+1}^{\bar{k}} t(k)\left(\left(1-\frac{w}{2}\right)^{k}-\frac{(1-w)^{k}}{2}\right)\right] p } \\
& +\delta\left[t(\widehat{k})(1-q)\left(1+(1-w)^{\widehat{k}}-2\left(1-\frac{w}{2}\right)^{\widehat{k}}\right)+\sum_{k=\widehat{k}+1}^{\bar{k}} t(k)\left(1+(1-w)^{k}-2\left(1-\frac{\widehat{w}}{2}\right)^{k}\right)\right](1-F(p)) p,
\end{aligned}
$$

where, in the square brackets in the first line, we have the share of consumers who do not compare prices and in the square brackets in the second line, we have the share of consumers who compare prices.

Equating this expression to the profit that the firm expects to make by charging $\bar{p}$, we can derive the equilibrium pricing distribution function:

$$
F(p)=1+\widehat{\eta}-\widehat{\eta} \frac{\bar{p}}{p}, \text { with support }[\underline{p}, \bar{p}]
$$

where (similar to the previous section) $\widehat{\eta}$ is the fraction of the share of consumers who do not compare prices to the share of consumers who compare prices. As before, the upper bound of the distribution is not larger than the reservation price $r$ and $v$, where the reservation price of a consumer is determined in (1) and $F(p)$ is given by (9).

Obviously, a full equilibrium analysis under asymmetric information on network structure is somewhat tedious. For a given $\widehat{k}$ and a $q(\widehat{k})$ one can derive the equilibrium price distribution, but given the equilibrium price distribution one should check whether the postulated behavior of consumers is indeed optimal and all consumers with $k>\widehat{k}$ prefer to be passive and all consumers with $k<\widehat{k}$ prefer to be active.

The proposition below states the main result for small enough values of $s$ :

Proposition 7 For any given $0 \leq t(1)<1$ and $0<\delta<1$, and sufficiently small search cost $s$, there exists an RPE given by the triple $(q, r, F(p))$ and the cutoff $\widehat{k}$, which are

\footnotetext{
${ }^{17}$ Unfortunately, we cannot use probability generating functions to simplify this expression further due to the fact that consumers search differently depending on the number of their connections.
} 
determined by (1), (9), and

$\frac{s}{v} \leq \frac{1-\delta}{1-\delta(1-w)^{\widehat{k}}+\frac{\widehat{\eta}}{1-\widehat{\eta} \ln \left(1+\frac{1}{\eta}\right)}\left[\delta\left(1+(1-w)^{\widehat{k}}-2\left(1-\frac{w}{2}\right)^{\widehat{k}}\right)\left((1+2 \widehat{\eta}) \ln \left(\frac{1+\widehat{\eta}}{\widehat{\eta}}\right)-2\right)+(1-\delta) \ln \left(\frac{1+\widehat{\eta}}{\hat{\eta}}\right)\right]}$,

where the inequality holds with equality if $0<q(\widehat{k})<1$. Furthermore, as $s \rightarrow 0$, the cutoff number of links $\widehat{k}$ equals $\bar{k}$ and the optimal search probability $q$ converges to 1 , while price dispersion disappears with $\bar{p}=\underline{p}=v$.

Figure 8 provides an illustrative example of the Proposition. ${ }^{18}$ Note that on the horizontal axis we represent $w$, the probability a random neighbor is active. The solid line representing the expected benefit from search has vertical elements. This is a consequence of the fact that for certain ranges of search costs, there is no indifferent consumer so that $w$, and the underlying decision of consumers whether or not to become active, does not change. This can be explained as follows. Suppose that the search cost is such that consumers with $\widehat{k}<\bar{k}$ links search with strictly positive probability, $0<q(\widehat{k})<1$. This is a situation represented by one of the non-vertical parts of the stable, right-hand side of the Figure. From Proposition 6, it follows that consumers with more than $\widehat{k}$ connections still search with probability one. Suppose then that the search cost decreases gradually. This change in search cost first affects the equilibrium search probability $q(\widehat{k})$ of consumers with $\widehat{k}$ connections, until this search probability obtains an extreme value of 1 . At this point, consumers with $\widehat{k}$ connections are indifferent between searching and not searching only when they search with probability one, while those with more than $\widehat{k}$ connections still search with probability zero. The difference in payoffs from being passive between consumers with $\widehat{k}$ friends and those with $\widehat{k}+1$ friends is related to the number of connections they have. This means that these two types of consumers cannot be indifferent between searching and not searching simultaneously. Then, obviously there is a range of search costs where consumers with $\widehat{k}$ connections strictly prefer to search, whereas those with $\widehat{k}+1$ links strictly prefer not to search (yet).

We do not explicitly perform the comparative statics exercise for this asymmetric model of search and information acquisition, as it is similar to the one in the previous section. In particular, it remains true that for any positive (sufficiently) small cost $s$ price

\footnotetext{
${ }^{18}$ The Figure is drawn for the following parameter values: $v=1, s=0.025, \delta=0.92, \bar{k}=5$, and $\gamma=-2.5$.
} 


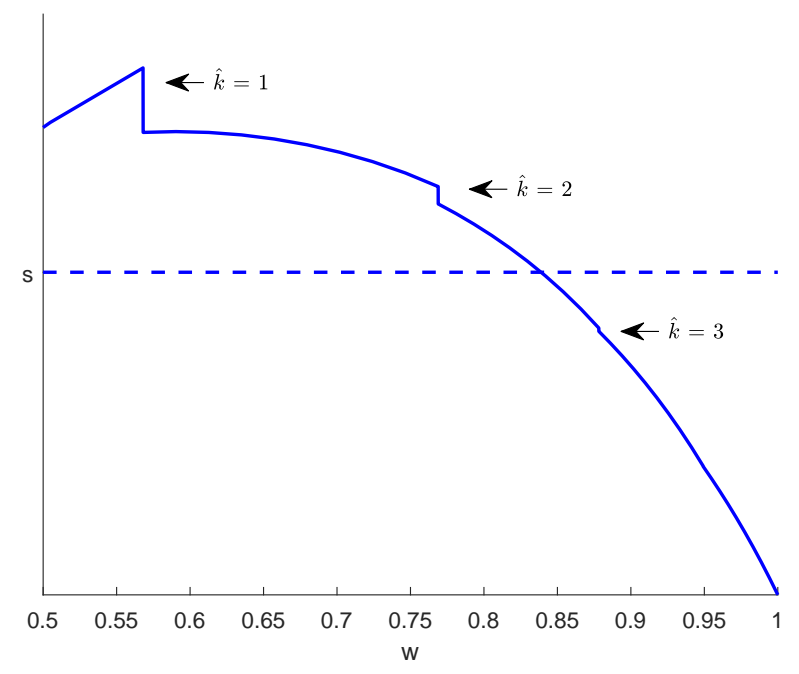

Figure 8: Illustration of existence of an RPE when consumers observe the number of direct links

dispersion remains if the number of connections becomes large as some consumers will be free riding.

\section{Conclusion}

In this paper we have analyzed how word-of-mouth (WOM) communication through social networks affects information acquisition and diffusion by consumers and how this impacts the market power of firms. Without WOM communication our model is prone to the Diamond paradox where the market breaks down due to the fact that no consumer makes price comparisons. WOM communication overcomes the Diamond paradox. Consumers that do not actively search themselves and free-ride on their friends in the social network may well be informed about different prices. The price comparisons they make provide positive externalities to the rest of the consumer population that actively searches as firms compete to be able to also sell to them.

As some consumers do compare prices, while others do not, the market is characterized by price dispersion. The level of prices and the nature of price dispersion depends on the network architecture, the search cost and how quickly information is diffused in the social network. In the context of evaluating the impact of online markets and social networks it is important to know how expected price, price dispersion and firms' profits react to $(i)$ a decrease in search cost, $(i i)$ an increase in the connectivity of the social network and 
(iii) an increase in the speed of information diffusion in the network. We find that there are opposing effects as the increased connectivity and speed of information diffusion lower expected market prices, whereas the decrease in search cost increases them. Importantly, price dispersion does not disappear even if all consumers are very well connected.

We see our paper as making a first step in analyzing how WOM communication and sequential search interact with each other. There are obvious ways our work can be extended in different directions. One direction that may be taken is to analyze markets with product differentiation a la Wolinsky (1986). In this case, consumers may not only communicate about prices, but also about the product match. In such markets, the degree of homophily (defined as the closeness of a consumer's preferences to those of his neighbors) will be important. Another direction for future research would be to model the incentives to share information directly. In some markets, consumers receive a financial benefit from firms for a successful referral and an important question is how consumers will react to such incentives and when such a financial incentive is optimal from a firm's perspective and to whom to give it. 


\section{Appendix A: Proofs}

\section{Proof of Theorem 1}

We use the following facts to rewrite the consumer's indifference condition. First, $E[p]=$ $\bar{p}-\int_{\underline{p}}^{\bar{p}} F(p) d p=\eta \bar{p} \ln \left(1+\frac{1}{\eta}\right)$. Second, $\bar{p}=r=\frac{s}{1-\eta \ln \left(1+\frac{1}{\eta}\right)}$. Third, $E\left[\min \left\{p_{1}, p_{2}\right\}\right]=$ $\bar{p}-2 \int_{\underline{p}}^{\bar{p}} F(p) d p+\int_{\underline{p}}^{\bar{p}} F^{2}(p) d p$ so that

$$
\begin{aligned}
E[p]-E\left[\min \left\{p_{1}, p_{2}\right\}\right] & =\int_{\underline{p}}^{\bar{p}} F(p) d p-\int_{\underline{p}}^{\bar{p}} F^{2}(p) d p \\
& =\eta \bar{p}\left((1+2 \eta) \ln \left(1+\frac{1}{\eta}\right)-2\right) .
\end{aligned}
$$

Thus, the consumers' indifference condition can be rewritten as

$$
\begin{aligned}
(1-\delta) v= & (1-\delta \tau(1-q)) s+\delta \widetilde{\tau}(q) \frac{\eta\left((1+2 \eta) \ln \left(1+\frac{1}{\eta}\right)-2\right)}{1-\eta \ln \left(1+\frac{1}{\eta}\right)} s \\
& +(1-\delta) s \frac{\eta \ln \left(1+\frac{1}{\eta}\right)}{1-\eta \ln \left(1+\frac{1}{\eta}\right)}
\end{aligned}
$$

or

$$
\frac{s}{v}=\frac{1-\delta}{1-\delta \tau(1-q)+\frac{\eta}{1-\eta \ln \left(\frac{1+\eta}{\eta}\right)}\left[\delta \widetilde{\tau}(q)\left((1+2 \eta) \ln \left(\frac{1+\eta}{\eta}\right)-2\right)+(1-\delta) \ln \left(\frac{1+\eta}{\eta}\right)\right]},
$$

We know that $\eta$ is a function of $q$ and that $\lim _{q \uparrow 1} \eta(q)=\infty$.

As the RHS of (10) is continuous in $0<q<1$, to prove the existence of an RPE for small enough values of $s$ it is sufficient to show that the RHS of (10) is positive and approaches 0 zero as $q \uparrow 1$. As $\eta \ln \left(\frac{1+\eta}{\eta}\right)<1$, the denominator is clearly positive if $\ln \left(\frac{1+\eta}{\eta}\right)>\frac{2}{1+2 \eta}$. As for $\eta \downarrow 0$ this latter inequality clearly holds, while the LHS and the RHS both approach 0 as $\eta \rightarrow \infty$, this inequality holds for all $\eta$ if the derivative of the LHS is more negative than that of the RHS. The derivate of the LHS is $-\frac{1}{\eta(1+\eta)}$, while the derivate of the RHS is $-\frac{2}{(1+2 \eta)^{2}}$. It is easy to see that the former derivate is smaller than the latter. Thus, as both numerator and denominator of (10) are positive, the whole expression is clearly positive.

Also that $1-\delta \leq 1-\delta \tau(1-q)$ means that the numerator is smaller than the denominator of the RHS of (10) for $0<q<1$. Thus, there exists an upper bound $\bar{s}$ on the search cost for which an RPE may exist.

To demonstrate the RHS of (10) converges to zero as $q \rightarrow 1$, we employ the following 
evaluations:

$$
\begin{gathered}
\lim _{\eta \rightarrow \infty} \eta \ln \left(1+\frac{1}{\eta}\right)=\lim _{z \downarrow 0} \frac{\ln (1+z)}{z} \stackrel{1^{\prime} \text { Hopital }}{=} \lim _{z \downarrow 0} \frac{1}{1+z}=1, \\
\lim _{\eta \rightarrow \infty} \frac{\eta \ln \left(1+\frac{1}{\eta}\right)}{1-\eta \ln \left(1+\frac{1}{\eta}\right)}=\frac{\lim _{\eta \rightarrow \infty} \eta \ln \left(1+\frac{1}{\eta}\right)}{1-\lim _{\eta \rightarrow \infty} \eta \ln \left(1+\frac{1}{\eta}\right)}=\infty,
\end{gathered}
$$

and

$$
\begin{aligned}
\lim _{\eta \rightarrow \infty} \frac{\eta\left((1+2 \eta) \ln \left(\frac{1+\eta}{\eta}\right)-2\right)}{1-\eta \ln \left(\frac{1+\eta}{\eta}\right)} & =\lim _{z \downarrow 0} \frac{\frac{1}{z}\left(\left(1+\frac{2}{z}\right) \ln (1+z)-2\right)}{1-\frac{\ln (1+z)}{z}} \\
& =\lim _{z \downarrow 0} \frac{(2+z) \ln (1+z)-2 z}{z^{2}-z \ln (1+z)} \\
\stackrel{\text { l'Hopital }}{=} \lim _{z \downarrow 0} \frac{\ln (1+z)+\frac{2+z}{1+z}-2}{2 z-\ln (1+z)-\frac{z}{1+z}} & =\lim _{z \downarrow 0} \frac{(1+z) \ln (1+z)-z}{z(1+2 z)-(1+z) \ln (1+z)} \\
& \stackrel{\text { l'Hopital }}{=} \lim _{z \downarrow 0} \frac{\frac{z}{\frac{(1+z)^{2}}{z(3+2 z)}}=\lim _{z \downarrow 0} \frac{1}{(1+z)^{2}}}{3+2 z}=\frac{1}{3} .
\end{aligned}
$$

Finally, noting $\lim _{q \uparrow 1} \widetilde{\tau}(q)=1-2 \tau(1 / 2)$, we can see that the denominator of the RHS of (10) increases unboundedly as $q \uparrow 1$. This means that the RHS of (10) converges to zero as $q \uparrow 1$.

Now, we show the limiting price for $s \downarrow 0$. We know that $s$ approaching zero is associated with $q \uparrow 1$, or $\eta \rightarrow \infty$ implying that price dispersion vanishes. Then, it suffices to evaluate the limiting value of $r$. We note that

$$
\begin{aligned}
r & =\frac{s}{1-\eta \ln \left(1+\frac{1}{\eta}\right)} \\
& =\frac{(1-\delta) v}{(1-\delta \tau(1-q))\left(1-\eta \ln \left(1+\frac{1}{\eta}\right)\right)+\eta\left[\delta \widetilde{\tau}(q)\left((1+2 \eta) \ln \left(\frac{1+\eta}{\eta}\right)-2\right)+(1-\delta) \ln \left(\frac{1+\eta}{\eta}\right)\right]} .
\end{aligned}
$$

Notice that the numerator of $r$ does not depend on $s$. Recalling (11), we can see that the 
first term in the denominator converges to zero as $q \uparrow 1$ (with associated $\eta \rightarrow \infty$ ). As

$$
\begin{aligned}
& \lim _{\eta \rightarrow \infty} \eta\left((1+2 \eta) \ln \left(\frac{1+\eta}{\eta}\right)-2\right)=\lim _{z \downarrow 0} \frac{\left(\left(1+\frac{2}{z}\right) \ln (1+z)-2\right)}{z} \\
&=\lim _{z \downarrow 0} \frac{((z+2) \ln (1+z)-2 z)}{z^{2}} \\
& \stackrel{\text { I'Hopital }}{=} \lim _{z \downarrow 0} \frac{\ln (1+z)+\frac{2+z}{1+z}-2}{2 z}=\lim _{z \downarrow 0} \frac{(1+z) \ln (1+z)-z}{2 z(1+z)} \\
& \stackrel{\text { ''Hopital }}{=} \lim _{z \downarrow 0} \frac{\ln (1+z)+1-1}{2+4 z}=0,
\end{aligned}
$$

it follows that the second term in the denominator approaches $1-\delta$ as $\eta \rightarrow \infty$. Then, the entire term converges to $(1-\delta) v /(1-\delta)=v$ as $q \uparrow 1$. This completes the proof.

\section{Proof of Proposition 2}

The proof that price dispersion must remain in the limit as $t(\infty) \rightarrow 1$ is by contradiction. Suppose to the contrary that price dispersion vanishes in the limit, or alternatively, that $\eta \rightarrow \infty$.

As

$$
\lim _{\eta \rightarrow \infty} r=\lim _{\eta \rightarrow \infty} \frac{s}{1-\eta \ln \left(1+\frac{1}{\eta}\right)}=\infty,
$$

it follows that in an RPE $E[p]$ must also increase without bound. But this would imply that the pay-off of becoming active is negative, which cannot be the case in active markets. Hence, price dispersion cannot vanish as $k \rightarrow \infty$ (or alternatively $t(\infty) \rightarrow 1$ ) and $\lim _{t(\infty) \rightarrow 1} q=\bar{q}$, where $0<\bar{q}<1$.

We next consider the question whether an active market exists in the limit as $t(\infty) \rightarrow$ 1. To this end, rewrite the consumers' indifference condition

$$
v-E[p]-s=\delta\left(v-E[p]+\widetilde{\tau}(q)\left(E[p]-E_{\min }[p]\right)-\tau(1-q) s\right),
$$

as

$$
\begin{aligned}
\frac{s}{v} & =\frac{1-\delta}{1-\delta \tau(1-q)+\left[\delta \widetilde{\tau}(q) \frac{E[p]-E_{\min }[p]}{s}+(1-\delta) \frac{E[p]}{s}\right]} \\
& =\frac{1-\delta}{1-\delta \tau(1-q)+\left[\frac{\eta}{1-\eta \ln \left(1-\frac{1}{\eta}\right)}\left\{\delta \widetilde{\tau}(q)\left((1+2 \eta) \ln \left(1+\frac{1}{\eta}\right)-2\right)+(1-\delta) \ln \left(1+\frac{1}{\eta}\right)\right\}\right]}
\end{aligned}
$$

where the LHS is the normalized cost of being active, while the RHS represents the incremental benefit of being so. Observe that the denominator of the RHS consists of three terms, the third one of which is the sum in the large square brackets. The first and the third terms are positive, while the second term is negative. As the second term converges to zero and $\widetilde{\tau}(q)$ to 1 (when $t(\infty) \rightarrow 1$ ), the limiting indifference condition is 
approximately

$$
\begin{aligned}
\frac{s}{v} & =\frac{1-\delta}{1+\frac{1}{s}\left[\lim _{t(\infty) \rightarrow 1} E[p]-\delta \lim _{t(\infty) \rightarrow 1} E_{\min }[p]\right]} \\
& =\frac{1-\delta}{1+\lim _{t(\infty) \rightarrow 1} \frac{\eta}{1-\eta \ln \left(1-\frac{1}{\eta}\right)}\left[\ln \left(1+\frac{1}{\eta}\right)-2 \delta\left(1-\eta \ln \left(1+\frac{1}{\eta}\right)\right)\right]}
\end{aligned}
$$

As the RHS approaches 0 if $\eta \rightarrow \infty$ it is clear this equation can always be satsified if $s$ is small enough.

\section{Proof of Proposition 3}

Observe that changes $s$ affect the LHS of (10) only. In particular, the LHS is increasing in $s$. As the RHS of the indifference equation (10) must be decreasing in $q$ in a stable RPE, the optimal search probability $q$ must be decreasing in $s$.

\section{Proof of Lemma 3}

Suppose that in equilibrium there is an atom at $\widetilde{p}$. As the share of active consumers is strictly positive, this price $\widetilde{p}$ gets compared to another price with strictly positive probability. As there is a strictly positive share of consumers that compare prices, undercutting $\widetilde{p}$ would be beneficial for firms as this yields a discontinuous increase in the demand, a contradiction. It follows that a firm that charges an upper bound $\bar{p}>r$ will not sell to any consumer.

\section{Proof of Lemma 6}

Observe that the LHS of (5) is strictly decreasing in $\rho_{1}$. Thus, if the RHS is increasing in $\rho$, then if a solution to (5) exists it must be a unique. From lemma 3, it follows that $F(p)$ must be a strictly increasing function of $p$ for $p \in\left[\underline{p}, \widetilde{p}_{1}\right]$ where $\underline{p}<\widetilde{p}_{1} \leq \min \{r, v\}$. Then, the RHS of (5) is strictly increasing in $\rho$ for $\rho \in\left[\underline{p}, \widetilde{p}_{1}\right]$ as

$$
F(\rho)(\rho-E[p \mid p<\rho])=F(\rho) \rho-\int_{\underline{p}}^{\rho} p d F(p)=\int_{\underline{p}}^{\rho} F(p) d p
$$

is strictly increasing in $\rho$. (Note that we applied integration by parts to obtain the last equality.) Next, suppose that $\widetilde{p}_{1}<\min \{r, v\}$ and $F(p)$ is flat in $p \in\left(\widetilde{p}_{1}, \widetilde{p}_{2}\right)$ where $\widetilde{p}_{1}<\widetilde{p}_{2} \leq \min \{r, v\}$. It means that $F(\rho)$ and $E[p \mid p<\rho]$ are constant for $\rho \in\left(\widetilde{p}_{1}, \widetilde{p}_{2}\right)$, but even then the RHS of (5) is increasing in $\rho \in\left(\widetilde{p}_{1}, \widetilde{p}_{2}\right)$. We can apply similar arguments to show that the RHS of (5) is increasing in $\rho$ for values of $\rho$ above $\widetilde{p}_{2}$ for shapes of $F(p)$ which have increasing and flat regions for $p \geq \widetilde{p}_{2}$. This proves that the RHS of (5) is indeed increasing in $\rho$, which in turn implies that if there is $\rho$ that satisfies (5) it must be unique. 


\section{Proof of Lemma 7}

Suppose to the contrary that $\rho<r$. First, notice that firms pricing at $\rho$ sell to all consumers who search them first, while if they price slightly above $\rho$, their demand drops as these consumers choose to wait for $p \in(\rho, r)$ and get informed of a lower price with a strictly positive probability, in which case they do not buy from the firm under question. Thus, if $F(\rho)<1$, there must exist a $\underline{r} \in(\rho, r)$ such that $F(\underline{r})=F(\rho)$. To simplify notation, let $E[p \leq \rho] \equiv E[p \mid p \leq \rho], E_{\min }[p \leq \rho] \equiv E\left[\min \left\{p_{1}, p_{2}\right\} \mid p_{1}, p_{2} \leq \rho\right], E[\underline{r} \leq p \leq$ $r] \equiv E[p \mid \underline{r} \leq p \leq r]$ and $E_{\min }[\underline{r} \leq p \leq r] \equiv E\left[\min \left\{p_{1}, p_{2}\right\} \mid \underline{r} \leq p_{1} \leq r, \underline{r} \leq p_{2} \leq r\right]$. If $F(\rho)=1$, we will say that $E[\underline{r} \leq p \leq r]=E_{\min }[\underline{r} \leq p \leq r]=r$. It follows that the reservation price $r$ is determined by

$$
\begin{aligned}
s & =r-E[p] \\
& =r-(1-F(\rho)) E[\underline{r} \leq p \leq r]-F(\rho) E[p \leq \rho] .
\end{aligned}
$$

As at price $r$ buyers at least weakly prefer waiting to buying, we can write that

$$
(1-\delta)(v-r)<\delta\left(1-\tau\left(1-\frac{q}{2}\right)\right) s .
$$

In equilibrium, an individual buyer is indifferent between being active and passive. The payoff from being active is

$$
\begin{array}{r}
F\left(\rho_{1}\right)\left(v-E\left[p \leq \rho_{1}\right]\right)+\delta\left(1-F\left(\rho_{1}\right)\right)(v-E[\underline{r} \leq p \leq r]) \\
+\delta\left(1-F\left(\rho_{1}\right)\right)^{2}\left(1-\tau\left(1-\frac{q}{2}\right)\right)\left(E[\underline{r} \leq p \leq r]-E_{\min }[\underline{r} \leq p \leq r]\right) \\
+\delta F\left(\rho_{1}\right)\left(1-F\left(\rho_{1}\right)\right)\left(1-\tau\left(1-\frac{q}{2}\right)\right)\left(E[\underline{r} \leq p \leq r]-E\left[p \leq \rho_{1}\right]\right)-s .
\end{array}
$$

Use (15) to expand the first term and simplify to obtain

$$
\begin{aligned}
F\left(\rho_{1}\right)(v-E[\underline{r} \leq & p \leq r])-(r-E[\underline{r} \leq p \leq r])+\delta\left(1-F\left(\rho_{1}\right)\right)(v-E[\underline{r} \leq p \leq r]) \\
& +\delta\left(1-F\left(\rho_{1}\right)\right)^{2}\left(1-\tau\left(1-\frac{q}{2}\right)\right)\left(E[\underline{r} \leq p \leq r]-E_{\min }[\underline{r} \leq p \leq r]\right) \\
& +\delta F\left(\rho_{1}\right)\left(1-F\left(\rho_{1}\right)\right)\left(1-\tau\left(1-\frac{q}{2}\right)\right)\left(E[\underline{r} \leq p \leq r]-E\left[p \leq \rho_{1}\right]\right) .
\end{aligned}
$$

Add and subtract $v$ and simplify to obtain

$$
\begin{array}{r}
v-r-(1-\delta)\left(1-F\left(\rho_{1}\right)\right)(v-E[\underline{r} \leq p \leq r]) \\
+\delta\left(1-F\left(\rho_{1}\right)\right)^{2}\left(1-\tau\left(1-\frac{q}{2}\right)\right)\left(E[\underline{r} \leq p \leq r]-E_{\min }[\underline{r} \leq p \leq r]\right) \\
+\delta F\left(\rho_{1}\right)\left(1-F\left(\rho_{1}\right)\right)\left(1-\tau\left(1-\frac{q}{2}\right)\right)\left(E[\underline{r} \leq p \leq r]-E\left[p \leq \rho_{1}\right]\right) .
\end{array}
$$


The payoff from being passive is

$$
\begin{array}{r}
\delta\left\{v-\left(1-F\left(\rho_{1}\right)\right)^{2}\left[E[\underline{r} \leq p \leq r]-\widetilde{\tau}(q)\left(E[\underline{r} \leq p \leq r]-E_{\min }[\underline{r} \leq p \leq r]\right)\right]\right. \\
-2 F\left(\rho_{1}\right)\left(1-F\left(\rho_{1}\right)\right)\left[\left(\tau\left(1-\frac{q}{2}\right)-\frac{\tau(1-q)}{2}\right) E[\underline{r} \leq p \leq r]+\left(1+\frac{\tau(1-q)}{2}-\tau\left(1-\frac{1}{2}\right)\right) E\left[p \leq \rho_{1}\right]\right] \\
\left.-F^{2}\left(\rho_{1}\right)\left[E\left[p \leq \rho_{1}\right]-\widetilde{\tau}(q)\left(E\left[p \leq \rho_{1}\right]-E_{\min }\left[p \leq \rho_{1}\right]\right)\right]-\tau(1-q) s\right\},
\end{array}
$$

or

$$
\begin{array}{r}
\delta\left\{v-\tau(1-q) s-\left(\left(1-F\left(\rho_{1}\right)\right)^{2}+2 F\left(\rho_{1}\right)\left(1-F\left(\rho_{1}\right)\right)\left(\tau\left(1-\frac{q}{2}\right)-\frac{\tau(1-q)}{2}\right)\right) E[\underline{r} \leq p \leq r]\right. \\
-\left(2 F\left(\rho_{1}\right)\left(1-F\left(\rho_{1}\right)\right)\left(1+\frac{\tau(1-q)}{2}-\tau\left(1-\frac{1}{2}\right)\right)+F^{2}\left(\rho_{1}\right)\right) E\left[p \leq \rho_{1}\right] \\
\left.+\widetilde{\tau}(q)\left(1-F\left(\rho_{1}\right)\right)^{2}\left(E[\underline{r} \leq p \leq r]-E_{\min }[\underline{r} \leq p \leq r]\right)+\widetilde{\tau}(q) F^{2}\left(\rho_{1}\right)\left(E\left[p \leq \rho_{1}\right]-E_{\min }\left[p \leq \rho_{1}\right]\right)\right\},
\end{array}
$$

Add and subtract $r$, use (15) to replace the positive $r$ and simplify to obtain

$$
\begin{array}{r}
\delta\{v-r+(1-\tau(1-q)) s \\
+\left(1-F\left(\rho_{1}\right)-\left(1-F\left(\rho_{1}\right)\right)^{2}-2 F\left(\rho_{1}\right)\left(1-F\left(\rho_{1}\right)\right)\left(\tau\left(1-\frac{q}{2}\right)-\frac{\tau(1-q)}{2}\right)\right) E[\underline{r} \leq p \leq r] \\
+\left(F\left(\rho_{1}\right)-2 F\left(\rho_{1}\right)\left(1-F\left(\rho_{1}\right)\right)\left(1+\frac{\tau(1-q)}{2}-\tau\left(1-\frac{1}{2}\right)\right)-F^{2}\left(\rho_{1}\right)\right) E\left[p \leq \rho_{1}\right] \\
\left.+\widetilde{\tau}(q)\left(1-F\left(\rho_{1}\right)\right)^{2}\left(E[\underline{r} \leq p \leq r]-E_{\min }[\underline{r} \leq p \leq r]\right)+\widetilde{\tau}(q) F^{2}\left(\rho_{1}\right)\left(E\left[p \leq \rho_{1}\right]-E_{\min }\left[p \leq \rho_{1}\right]\right)\right\},
\end{array}
$$

However, as the multiplicative terms of both $E[\underline{r} \leq p \leq r]$ and $\left(-E\left[p \leq \rho_{1}\right]\right)$ simplifies to $\widetilde{\tau}(q) F\left(\rho_{1}\right)\left(1-F\left(\rho_{1}\right)\right)$, we can rewrite the expression as

$$
\delta\left\{v-r+(1-\tau(1-q)) s+\widetilde{\tau}(q) F\left(\rho_{1}\right)\left(1-F\left(\rho_{1}\right)\right)\left(E[\underline{r} \leq p \leq r]-E\left[p \leq \rho_{1}\right]\right)\right.
$$

$\left.+\widetilde{\tau}(q)\left(1-F\left(\rho_{1}\right)\right)^{2}\left(E[\underline{r} \leq p \leq r]-E_{\min }[\underline{r} \leq p \leq r]\right)+\widetilde{\tau}(q) F^{2}\left(\rho_{1}\right)\left(E\left[p \leq \rho_{1}\right]-E_{\min }\left[p \leq \rho_{1}\right]\right)\right\}$.

Thus, the indifference condition of buyers is

$$
\begin{array}{r}
(1-\delta)(v-r)=\delta(1-\tau(1-q)) s+(1-\delta)\left(1-F\left(\rho_{1}\right)\right)(v-E[\underline{r} \leq p \leq r]) \\
-\delta F\left(\rho_{1}\right)\left(1-F\left(\rho_{1}\right)\right)\left(\tau\left(1-\frac{q}{2}\right)-\tau(1-q)\right)\left(E[\underline{r} \leq p \leq r]-E\left[p \leq \rho_{1}\right]\right) \\
-\delta\left(1-F\left(\rho_{1}\right)\right)^{2}\left(\tau\left(1-\frac{q}{2}\right)-\tau(1-q)\right)\left(E[\underline{r} \leq p \leq r]-E_{\min }[\underline{r} \leq p \leq r]\right) \\
+\delta \widetilde{\tau}(q) F^{2}\left(\rho_{1}\right)\left(E\left[p \leq \rho_{1}\right]-E_{\min }\left[p \leq \rho_{1}\right]\right)
\end{array}
$$


As

$$
\begin{array}{r}
(1-\tau(1-q)) s=\left(1-\tau\left(1-\frac{q}{2}\right)\right) s+\left(\tau\left(1-\frac{q}{2}\right)-\tau(1-q)\right) s \\
=\left(1-\tau\left(1-\frac{q}{2}\right)\right) s+\left(\tau\left(1-\frac{q}{2}\right)-\tau(1-q)\right)\left(r-E[\underline{r} \leq p \leq r]+F\left(\rho_{1}\right)\left(E[\underline{r} \leq p \leq r]-E\left[p \leq \rho_{1}\right]\right)\right),
\end{array}
$$

where we used (15) to obtain the second equality, we can rewrite the indifference condition as

$$
\begin{array}{r}
(1-\delta)(v-r)=\delta\left(1-\tau\left(1-\frac{q}{2}\right)\right) s+(1-\delta)\left(1-F\left(\rho_{1}\right)\right)(v-E[\underline{r} \leq p \leq r]) \\
+\delta F\left(\rho_{1}\right)^{2}\left(\tau\left(1-\frac{q}{2}\right)-\tau(1-q)\right)\left(E[\underline{r} \leq p \leq r]-E\left[p \leq \rho_{1}\right]\right) \\
+\delta \widetilde{\tau}(q)\left(\tau\left(1-\frac{q}{2}\right)-\tau(1-q)\right)\left(r-E[\underline{r} \leq p \leq r]-\left(1-F\left(\rho_{1}\right)\right)^{2}\left(E[\underline{r} \leq p \leq r]-E_{\min }[\underline{r} \leq p \leq r]\right)\right) \\
+\delta \widetilde{\tau}(q) F^{2}\left(\rho_{1}\right)\left(E\left[p \leq \rho_{1}\right]-E_{\min }\left[p \leq \rho_{1}\right]\right) .
\end{array}
$$

The LHS of the equation is equal to the LHS of (16). The RHS of the indifference equation is certainly strictly larger than the RHS of (16) as all the terms that are not included in (16) are nonnegative, while independent of whether $F(\rho)=1$ or $F(\rho)<1$ at least some terms are strictly positive. Thus, the indifference condition of consumers and (16) cannot hold simultaneously. This completes the proof that it cannot be that $\rho<r$.

\section{Proof of Proposition 5}

If we for the time being write $\frac{q}{2 \delta(1-q)}=\eta$, and using the expressions for $E[p]$ and $E_{\min }[p]$ that were gien in the proof of Theorem 1, we can rewrite the indifference condition (6) as

$$
\begin{aligned}
& (1-\delta)(v-E[p])-s=\delta\left(E[p]-E_{\min }[p]\right), \\
& (1-\delta)\left[v-\frac{\eta s \ln \left(1+\frac{1}{\eta}\right)}{1-\eta \ln \left(1+\frac{1}{\eta}\right)}\right]-s=\delta \frac{\eta s\left((1+2 \eta) \ln \left(1+\frac{1}{\eta}\right)-2\right)}{1-\eta \ln \left(1+\frac{1}{\eta}\right)}, \\
& (1-\delta) v-s=(1-\delta) \frac{\eta s \ln \left(1+\frac{1}{\eta}\right)}{1-\eta \ln \left(1+\frac{1}{\eta}\right)}+\delta \frac{\eta s\left((1+2 \eta) \ln \left(1+\frac{1}{\eta}\right)-2\right)}{1-\eta \ln \left(1+\frac{1}{\eta}\right)}, \\
& (1-\delta)=\frac{s}{v}\left[1+(1-\delta) \frac{\eta \ln \left(1+\frac{1}{\eta}\right)}{1-\eta \ln \left(1+\frac{1}{\eta}\right)}+\delta \frac{\eta\left((1+2 \eta) \ln \left(1+\frac{1}{\eta}\right)-2\right)}{1-\eta \ln \left(1+\frac{1}{\eta}\right)}\right]
\end{aligned}
$$

Further rewriting gives

$$
\begin{gathered}
\frac{(1-\delta)\left(1-\eta \ln \left(1+\frac{1}{\eta}\right)\right)}{1-\delta \eta \ln \left(1+\frac{1}{\eta}\right)+\delta \eta\left((1+2 \eta) \ln \left(1+\frac{1}{\eta}\right)-2\right)}=\frac{s}{v} \\
\frac{(1-\delta)\left(1-\eta \ln \left(1+\frac{1}{\eta}\right)\right)}{1-2 \delta \eta\left(1-\eta \ln \left(1+\frac{1}{\eta}\right)\right)}=\frac{s}{v}
\end{gathered}
$$


As the LHS is decreasing in $\eta$ and approaches 0 as $\eta \rightarrow \infty$, it follows that for small enough $s$, there exists a unique value of $\eta$ that soles the indifference condition. As $\eta$ is increasing in $q$ and $\eta \rightarrow \infty$ as $q$ approaches 1 , it follows that as $s \rightarrow 0, q \rightarrow 1$. The rest of the proof is identical to the proof of Theorem 1 and therefore omitted.

\section{Proof of Proposition 6}

We prove the proposition with the help of two claims.

Claim 1 If consumers with $1 \leq \widehat{k} \leq \bar{k}$ number of links search with strictly positive probability, all consumers with numbers of links less than $\widehat{k}$ links (if there are such) search with probability one.

Proof. As $w$ represents the probability a consumer assigns to a neighbor actively searching, a consumer with $\widehat{k}$ friends searches with positive probability only if doing so is weakly better than not searching, i.e.,

$\delta\left(v-E[p]+\left(1+(1-w)^{\widehat{k}}-2\left(1-\frac{w}{2}\right)^{\widehat{k}}\right)\left(E[p]-E_{\min }[p]\right)-(1-w)^{\widehat{k}} s\right) \leq v-E[p]-s$.

For consumers with less than $\widehat{k}$ links, searching yields the same payoff as the RHS of the inequality, whereas not searching yields a payoff strictly smaller than the LHS of the inequality as the LHS is increasing in $\widehat{k}$ for $0<w<1$. Hence, consumers with less than $\widehat{k}$ connections search for sure.

Claim 2 If consumers with $1 \leq \widehat{k} \leq \bar{k}$ number of links search with positive probability less than 1, all consumers with numbers of links greater than $\widehat{k}$ links (if there are such) do not search.

Proof. The proof is analogous to the proof of Claim 1.

If a consumer with $\widehat{k}$ links is indifferent between searching and not searching, her optimal search probability lies between zero and one. Then, from the above two claims it follows that consumers with lower than $\widehat{k}$ search for sure, whereas those with greater than $\widehat{k}$ links do not search at all.

\section{Proof of Proposition 7}

Some parts of the proof are similar to the proof of Theorem 1. To avoid repetition, we omit some details here. It is clear that to obtain the equilibrium distribution function in (9), we should have

$\widehat{\eta}=\frac{\frac{\widehat{w}}{2}+\delta t(\widehat{k})(1-q)\left(\left(1-\frac{w}{2}\right)^{\widehat{k}}-\frac{(1-w)^{\widehat{k}}}{2}\right)+\delta \sum_{k=\widehat{k}+1}^{\bar{k}} t(k)\left(\left(1-\frac{w}{2}\right)^{k}-\frac{(1-w)^{k}}{2}\right)}{\delta t(\widehat{k})(1-q)\left(1+(1-w)^{\widehat{k}}-2\left(1-\frac{w}{2}\right)^{\widehat{k}}\right)+\delta \sum_{k=\widehat{k}+1}^{\bar{k}} t(k)\left(1+(1-w)^{k}-2\left(1-\frac{\widehat{w}}{2}\right)^{k}\right)}$.

It is clear that $\widehat{\eta}$ is a function of $\widehat{k}$ and $q$ and that $\lim _{q \rightarrow 1} \widehat{\eta}(\widehat{k}=\bar{k}, q)=\infty$. 
If a consumer with $\widehat{k}$ friends is indifferent between being passive and active, then it must be the case that the pay-off of being passive

$$
\delta\left(v-E[p]+\left(1+(1-w)^{\widehat{k}}-2\left(1-\frac{w}{2}\right)^{\widehat{k}}\right)\left(E[p]-E_{\min }[p]\right)-(1-w)^{\widehat{k}} s\right)
$$

is equal to the pay-off $v-E[p]-s$ of being active. We can rewrite this indifference equation as

$$
\left(1-\delta(1-w)^{\widehat{k}}\right) s+\delta W\left(E[p]-E_{\min }[p]\right)=(1-\delta)(v-E[p])
$$

where $W=\left[1+(1-w)^{\widehat{k}}-2\left(1-\frac{w}{2}\right)^{\widehat{k}}\right]$ and, using the expressions for $E[p], E[p]-E_{\min }[p]$ and $\bar{p}$ developed in the beginning of the proof of Theorem 1, express this condition further as

$$
\left(1-\delta(1-w)^{\widehat{k}}\right) s+s \delta W \frac{\widehat{\eta}\left((1+2 \widehat{\eta}) \ln \left(1+\frac{1}{\widehat{\eta}}\right)-2\right)}{1-\widehat{\eta} \ln \left(1+\frac{1}{\hat{\eta}}\right)}=(1-\delta)\left(v-\frac{\widehat{\eta} \ln \left(1+\frac{1}{\widehat{\eta}}\right)}{1-\widehat{\eta} \ln \left(1+\frac{1}{\bar{\eta}}\right)} s\right)
$$

Bringing all the terms with $s$ on one side and re-arranging gives the condition mentioned in the Proposition.

We now show that if $s \rightarrow 0, \widehat{k}=\bar{k}$ and $q(\widehat{k}) \uparrow 1$ hold in equilibrium. It is clear that if $s \rightarrow 0$ price dispersion must disappear as otherwise active consumers would have an incentive to continue searching. Suppose then that if $s \rightarrow 0, \widehat{k}<\bar{k}$. This would imply that for consumers with $\bar{k}$ friends the pay-off of waiting is strictly larger than the pay-off of actively searching. However, with price dispersion disappearing if $s \rightarrow 0$, for all $0<\delta<1$ and for every consumer (no matter how many friends she has) this cannot be the case. If $q(\widehat{k})$ would not converge to 1 if $s \rightarrow 0$, then $\widehat{\eta}$ would converge to a finite number and price dispersion would persist, a contradiction.

Finally, we focus on the price level to which the price distribution converges if $s \rightarrow 0$. As $\widehat{k}=\bar{k}, \widehat{\eta}$ reduces to

$$
\widehat{\eta}=\frac{\frac{\widehat{w}}{2}+\delta t(\widehat{k})(1-q)\left(\left(1-\frac{w}{2}\right)^{\widehat{k}}-\frac{(1-w)^{\widehat{k}}}{2}\right)}{\delta t(\widehat{k})(1-q) W}
$$

so that $\lim _{q \uparrow 1} \widehat{\eta}=\infty$ as both $w$ and $\widehat{w}$ converge to 1 . Since search behavior of consumers with $\bar{k}$ links is of interest in the limit, we can write

$$
\begin{aligned}
& \lim _{s \rightarrow 0} r=\lim _{s \rightarrow 0} \frac{s}{1-\widehat{\eta} \ln \left(\frac{1}{\hat{\eta}}+1\right)} \\
& =\lim _{q \uparrow 1} \frac{(1-\delta) v}{\left(1-\delta(1-w)^{\widehat{k}}\right)\left(1-\widehat{\eta} \ln \left(1+\frac{1}{\hat{\eta}}\right)\right)+\widehat{\eta}\left[\delta W\left((1+2 \widehat{\eta}) \ln \left(\frac{1+\widehat{\eta}}{\hat{\eta}}\right)-2\right)+(1-\delta) \ln \left(\frac{1+\widehat{\eta}}{\widehat{\eta}}\right)\right]} .
\end{aligned}
$$

To evaluate the limit, we undertake similar steps as when we evaluated the limiting $r$ in the proof of Theorem 1. Note that the numerator of the expression is independent of 
q. In the denominator, the first two term converges to zero, following (11). The second term in the denominator converges to $1-\delta$ since

$$
\begin{aligned}
& \lim _{\widehat{\eta} \rightarrow \infty} \widehat{\eta}\left((1+2 \widehat{\eta}) \ln \left(\frac{1+\widehat{\eta}}{\widehat{\eta}}\right)-2\right)=\lim _{z \downarrow 0} \frac{\left(\left(1+\frac{2}{z}\right) \ln (1+z)-2\right)}{z} \\
&=\lim _{z \downarrow 0} \frac{((z+2) \ln (1+z)-2 z)}{z^{2}} \\
& \stackrel{\text { I'Hopital }}{=} \lim _{z \downarrow 0} \frac{\ln (1+z)+\frac{2+z}{1+z}-2}{2 z}=\lim _{z \downarrow 0} \frac{(1+z) \ln (1+z)-z}{2 z(1+z)} \\
& \stackrel{\text { l'Hopital }}{=} \lim _{z \downarrow 0} \frac{\ln (1+z)+1-1}{2+4 z}=0 .
\end{aligned}
$$

Summing up, the limiting $r$ converges to $\frac{(1-\delta) v}{1-\delta}=v$. The proof is complete. 


\section{References}

Aker, Jenny, "Information from Markets Near and Far: Mobile Phones and Agricultural Markets in Niger," American Economic Journal: Applied Economics, 2010, 2 (3), 4659.

_ and Isaac Mbiti, "Mobile Phones and Economic Development in Africa," Journal of Economic Perspectives, 2010, 24 (3), 207-232.

Arbatskaya, Maria and Hideo Konishi, "Consumer Referrals," International Journal of Industrial Organization, 2016, 48, 34-58.

Atayev, Atabek and Maarten Janssen, "Information Acquisition and Diffusion in Markets," CEPR Discussion Paper DP14036, 2019.

Banerji, Abhijit and Bhaskar Dutta, "Local Network Externalities and Market Segmentation," International Journal of Industrial Organization, 2009, 27, 605-614.

Biyalogorsky, Eyal, Eitan Gerstner, and Barak Libai, "Customer Referral Management: Optimal Reward Programs," Marketing Science, 2001, 20 (1), 82-95.

Bloch, Francis, "Targeting and Pricing in Social Networks," in Yann Bramoulle, Andrea Galeotti, and Brian Rogers, eds., Oxford Handbook of the Economics of Networks, Oxford: Oxford University Press, 2016.

Bramoulle, Yann and Rachel Kranton, "Public Goods in Networks," Journal of Economic Theory, 2007, 135, 478-494.

Brown, Jeffrey and Austan Goolsbee, "Does the Internet Make Markets More Competitive? Evidence from the Life Insurance Industry," Journal of Policitcal Economy, 2002, $110(3), 481-507$.

Burdett, Kenneth and Kenneth Judd, "Equilibrium Price Dispersion," Econometrica, 1983, 51 (4), 955-969.

Campbell, Arthur, "Word-of-Mouth Communication and Percolation in Social Networks," American Economic Review, 2013, 103 (6), 2466-2498. 
_ , "Social Learning with Differentiated Products," RAND Journal of Economics, 2019, $50,226-248$.

_, Matthew Leister, and Yves Zenou, "Word-of-Mouth Communication and Search," RAND Journal of Economics, 2020, 51 (3), 676-712.

Chen, Ying-Ju, Yves Zenou, and Junjie Zhou, "Competitive Pricing Strategies in Social Networks," RAND Journal of Economics, 2018, 49, 672-705.

Chen, Yubo, Qu Wang, and Jinhong Xie, "Online Social Interactions: A Natural Experiment on Word of Mouth Versus Observational Learning," Journal of Marketing Research, 2011, 48 (2), 238-254.

Chuhay, Roman, "Pricing Innovation in the Presence of Word-of-Mouth Communication," Working Paper, April 2015.

Diamond, Peter, "A Model of Price Adjustment," Journal of Economics Theory, 1971, 3, 156-168.

Ellison, Glenn and Drew Fudenberg, "Word-of-Mouth Communication and Social Learning," The Quarterly Journal of Economics, 1995, 110, 93-125.

Fainmesser, Itay and Andrea Galeotti, "Pricing Network Effects," Review of Economic Studies, 2016, 83, 165-198.

_ and _, "Pricing Network Effects: Competition," American Economic Journal: Microeconomics, 2020, forthcoming.

Fershtman, Chaim and Arthur Fishman, "Price Cycles and Booms: Dynamic Search Equilibrium," American Economic Review, 1992, 82 (5), 1221-1233.

Galeotti, Andrea, "Talking, Searching, and Pricing," International Economic Review, 2010, 51 (4), 1159-1174.

- and Sanjeev Goyal, "Influencing the Influencers: a Theory of Strategic Diffusion," RAND Journal of Economics, 2009, 40 (3), 509-532.

_ and _, "The Law of the Few," American Economic Review, 2010, 100 (4), 1468-1492. 
Garcia, Daniel and Sandro Shelegia, "Consumer Search with Observational Learning," RAND Journal of Economics, 2018, 49, 224-253.

Godes, David and Dina Mayzlin, "Using Online Conversations to Study Word-ofMouth Communication," Marketing Science, 2004, 23 (4), 545-560.

Grossman, Sanford J. and Joseph E. Stiglitz, "On the Impossibility of Informationally Efficient Market," American Economic Review, 1980, 70 (3), 393-408.

Hayek, Friedrich, "The Use of Knowledge in Society," American Economic Review, 1945, $35(4), 519-530$.

Honda, Jun, "Intermediary Search for Suppliers in Procurement Auctions," Working Paper, 2015.

Jackson, Matthew, Social and Economic Networks, Princeton University Press, 2008.

Janssen, Maarten and Jose Luis Moraga-Gonzalez, "Strategic Pricing, Consumer Search and the Number of Firms," Review of Economic Studies, 2004, 71, 1089-1118.

_ , _ , and Matthijs R. Wildenbeest, "Truly Costly Sequential Search and Oligopolistic Pricing," International Journal of Industrial Organization, 2005, 23, 451-466.

Jensen, Robert, "The Digital Provide: Information (Technology), Market Performance, and Welfare in South Indian Fisheries Sectior," The Quarterly Journal of Economics, 2007, CXXII, 879-924.

Jun, Tackseung and Jeong-Yoo Kim, "A Theory of Consumer Referral," International Journal of Industrial Organization, 2008, 26, 662-678.

Katz, Elihu and Paul Felix Lazarsfeld, Personal Influence, New York: Free Press, 1955.

Kircher, Philipp and Andrew Postlewaite, "Strategic Firms and Endegenous Consumer Emulation," The Quarterly Journal of Economics, 2008, 123, 621-661.

Kohn, Meir and Steven Shavell, "The Theory of Search," Journal of Economic Theory, 1974, 9, 93-123. 
Kornish, Laura and Qiuping Li, "Optimal Referral Bonuses with Asymmetric Information: Firm-Offered and Interpersonal Incentives," Marketing Science, 108-121 2010, $29(1), 108-121$.

Miegielsen, Simon, "Consumer Information Networks," KU Leuven Discussion Paper Series. DPS14.14, 2014.

Price, Derek, "Networks of Scientific Papers," Science, 1965, 149 (3683), 510-515.

Seiler, Stephan, Song Yao, and Wenbo Wang, "Does Online Word of Mouth Increase Demand? (And How?) Evidence from a Natural Experiment," Marketing Science, 2019, $36(6), 838-861$.

Stahl, Dale O.II, "Oligopolistic Pricing with Sequential Consumer Search," American Economic Review, September 1989, 79 (4), 700-712.

Stigler, George, "The Economics of Information," Journal of Political Economy, 1961, $69(3), 213-225$.

Varian, Hal R., "A Model of Sales," American Economic Review, September 1980, 70 (4), 651-659.

Wolinsky, Asher, "True Monopolistic Competition as a Result of Imperfect Information," Quarterly Journal of Economics, August 1986, 101 (3), 493-512. 